\title{
A brief Review of $C_{n}$ symmetric Calixarenes and Resorcinarenes
}

\author{
M.J. McIldowie, M. Mocerino and M.I. Ogden \\ Dept. of Applied Chemistry, Curtin University of Technology, Perth, Australia.
}

\begin{abstract}
Calixarene and resorcinarene macrocycles are renowned for their ability to form inclusion complexes or act as molecular scaffolds. The addition of chirality to these non-planar molecules is an exciting enhancement of their already robust potential, offering much promise as ligands for chiral catalysis and enantioselective separations. Chiral calixarenes can be produced by the attachment of a chiral moiety or by the placement of an achiral functionality on the macrocyclic structure so as to render it asymmetric. The latter method is particularly intriguing, often resulting in molecules which have $C_{\mathrm{n}}$ dissymmetry. This review describes examples of the $C_{\mathrm{n}}$ dissymmetric calixarenes and resorcinarenes prepared to date and discusses aspects of their chirality, including their pictorial and written descriptors.
\end{abstract}

Keywords: calixarene, resorcinarene, chirality, dissymmetry

\section{Introduction}

Calixarenes and resorcinarenes have been successfully applied in a variety of roles such as catalysis 1 , as selective ionophores2 and chromatographic stationary phases3. The addition of chirality to these non-planar molecules not only offers great potential for the enhancement of these applications but also for the development of exciting new functionality.

Chiral calixarenes can be categorised into two distinct subtypes, each with their own distinct methods of preparation. The categories are commonly described as chiral by attachment and chiral by asymmetric functionalisation. The factor separating these two groups is unmistakably the method by which the chirality of the calixarene is generated.

Chirality by attachment, as the name suggests, is simply the generation of a chiral calixarene or resorcinarene species by appending an already chiral functionality to the macrocyclic skeleton. This is by far the easiest method for preparing an enantiomerically pure calixarene species by virtue of the fact that no resolution 
procedure is required and there are many chiral materials available as single enantiomers. Many of these materials also have some useful functionality that may be used to attach them to the calixarene skeleton. Consequently, when combined with the various positions on the macrocyclic framework available for attachment, there are numerous possible combinations for the calixarene chemist to explore. Two admirable examples are the upper rim amino-acid functionalised compound (A)

(Figure 1 (A)) produced by Ungaro4 and lower rim sugar functionalised compound (B) (Figure 1 (B)) produced by Lhoták.5

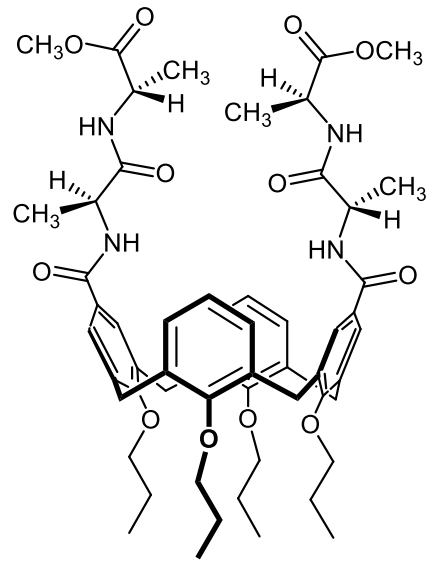

(A)

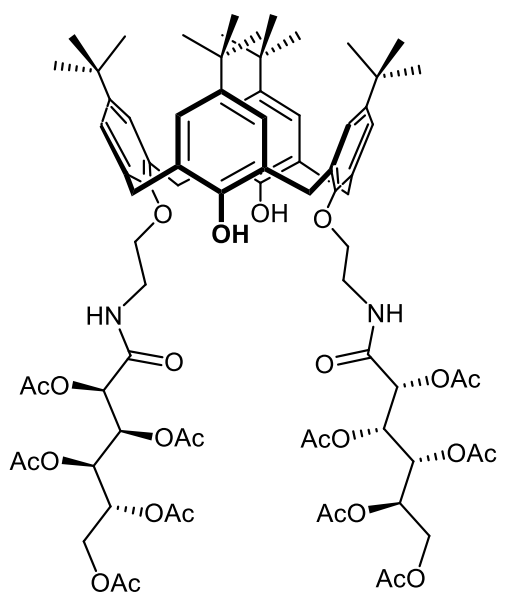

(B)

Figure 1. Two examples of chiral calixarenes formed by the attachment of a chiral moiety.

Chirality by asymmetric functionalisation essentially encompasses the remainder of the chiral calixarenes. A broad definition for this class would simply be generation of chirality by functionalisation of the non-planar calixarene framework with achiral moieties. This is achieved by attachment of functionality at particular positions so as to destroy the $C_{4 \mathrm{v}}$ symmetry of the calixarenes and resorcinarenes and thus afford the lower symmetry $C_{2}$ or $C_{1}$ symmetric entities. This subcategory can be divided into two further groups which are separated predominantly by symmetry concerns. The first group encompasses macrocycles which have been made chiral by the introduction of achiral substituents at the phenols or at the $\mathrm{X}_{5 \dagger} \uparrow$ positions in a manner 
so as to render the molecule asymmetric. These molecules have no mirror planes or symmetry axes (greater than $C_{1}$ ) and can be described as "planar chiral" or "conformationally chiral". 7 For example a calixarene such as that shown in figure 2 having four differently substituted aromatic units must necessarily be an asymmetric molecule. In fact it can be stated that in general in order to produce this type of asymmetry a minimum of two functionalisations on the calixarene is required to produce three adjacent aromatic units which are different. The remaining aromatic unit in this case may be anything except B (A, C or D). Thus the aromatic units in a chiral calixarene of this type may be of the pattern $\mathrm{ABCD}$ or $\mathrm{AABC}$.
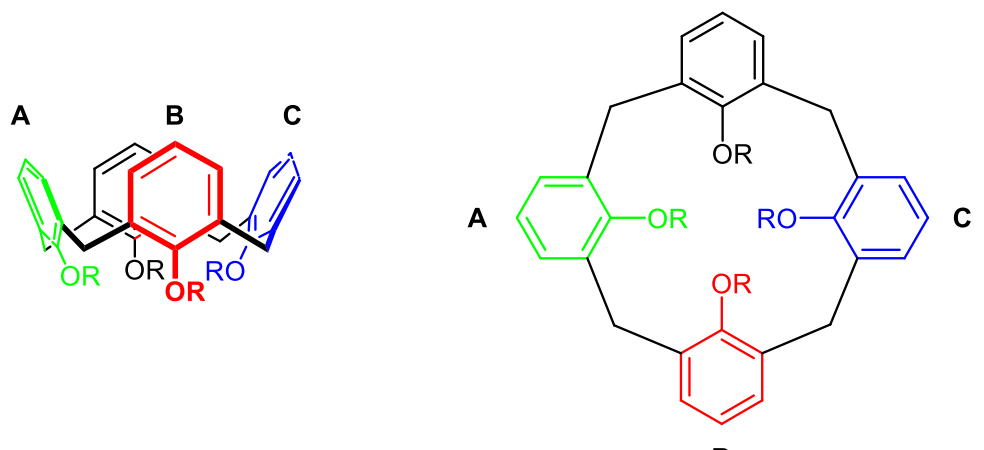

B

Figure 2. Side and top views of a chiral calixarene having three sequential different aromatic units.

The second form of asymmetric functionalisation is comprised of those molecules for which asymmetry has be generated by the inclusion of a group at the $\mathrm{X}_{4}$ or $\mathrm{X}_{6}$ positions (or both) which also destroys the vertical mirror planes of the $C 4 \mathrm{v}$ macrocycles. This is shown in figure 3 where the position of $\mathrm{R}_{1}$ (in the case of the calixarene $\mathbf{A}$ ) and the kind of functionality at $\mathrm{R}_{2}$ (in the case of the resorcinarene $\mathbf{B}$ ) renders the desired asymmetry.

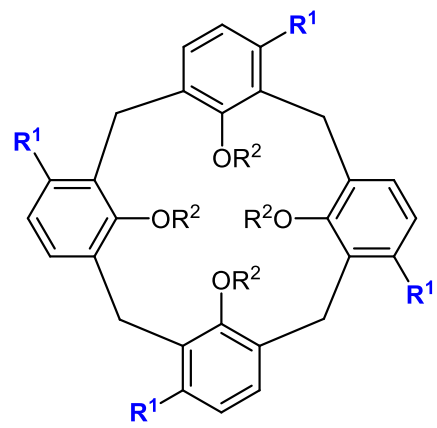

(A)

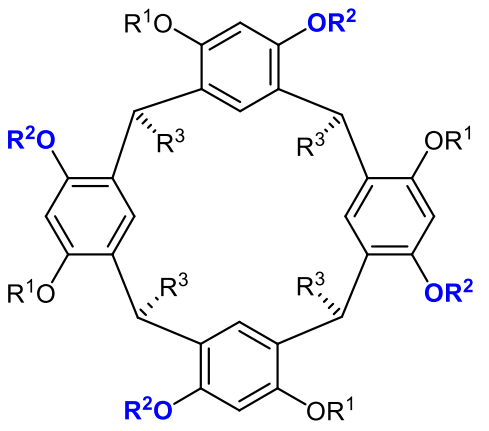

(B) 
Figure 3. Examples of a chiral dissymmetric calixarene (A) (where $\mathrm{R}_{1} \neq \mathrm{H}$ ) and chiral dissymmetric resorcinarene $(\mathbf{B})\left(\right.$ where $\left.\mathrm{R}_{1} \neq \mathrm{R}_{2}\right)$.

In these cases only a single different functionality at the $\mathrm{X}_{4}$ or $\mathrm{X}_{6}$ positions generates the desired asymmetry, unlike the AABC type (see Figure 2) where at least two different functionalisations are required.:

Elements of conformational, axial or helical chirality may potentially be ascribed to calixarene (A) (Figure 3). The "chirality axis" (co-incident with the $C_{\text {n }}$ symmetry axis) has generally been favoured in the calixarene literature, although, a proposal for modification of the Cahn, Ingold and Prelog classification of chirality recommended the usage of the "helical axis".8 The term "inherent chirality" 9 is often applied but has no stereochemical significance 10 and has more suitable replacements in the stereochemical literature 11. Application of these chirality descriptors to calix[4]arenes is commonplace, however, despite the abundance of completely asymmetric calix[4]arenes (for examples see references 12,13) with an AABC type pattern, no formal method for describing their absolute stereogenicity appears to have been applied. In 2004, Mandolini proposed a method for describing the chirality sense of the AABC type calixarene derivatives 10 (Figure 4). By assigning a priority order ( $a, b, c$ and $d)$ to the calixarene methylenes, an observer standing within the calixarene cavity could then demonstrate a clockwise $(\mathrm{c} R)$ or counterclockwise $(\mathrm{c} S)$ array.

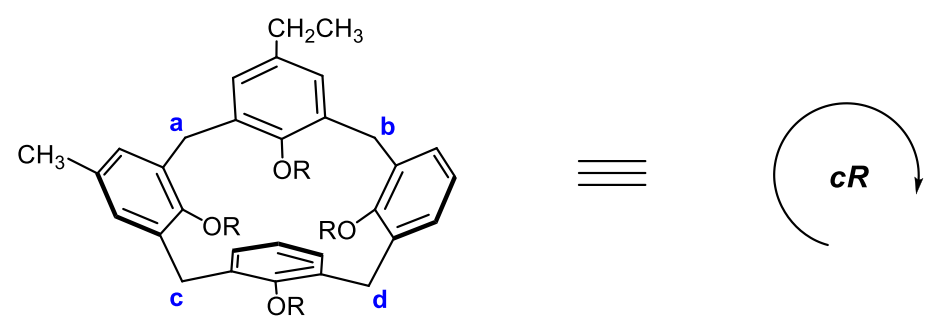

Figure 4. Application of Mandolini's priority method for assignment of chirality sense. 
However, the majority of calixarene publications (for example14), have not adopted this notation since it was introduced, with a few exceptions.15-17

Similarly untested are the rules delineated by Prelog and Helmchen for describing the chirality sense of those molecules not containing any stereogenic centres 18 i.e. those having a "chirality plane" or a "chirality axis". This method was exemplified in 1984 by Schlögl for smaller phanes19 such as the benzoic acid derivative (A) given in

\section{figure 5.}

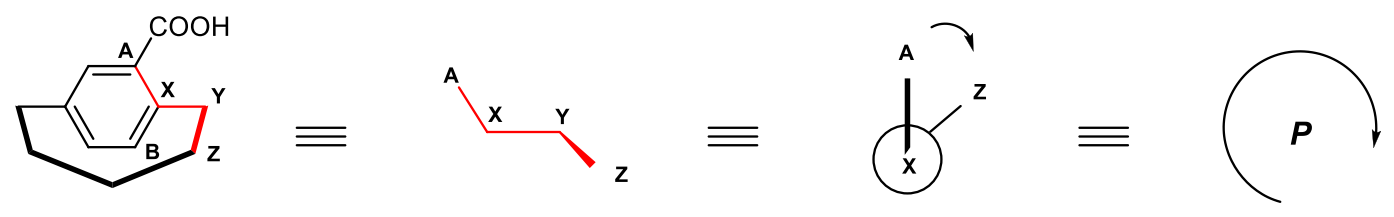

(A)

Figure 5. Application of the helical chirality descriptor to phane molecules.

Description of resorcinarene chirality on the other hand has no such complications as the presence of four stereogenic centres makes application of the IUPAC prescribed 20 Cahn-Ingold-Prelog rules7 a simple task. Thus the structures A and B in figure 6 below can be described as the $2 R, 4 R, 6 R, 8 R$ - and $2 S, 4 S, 6 S, 8 S$ - respectively.

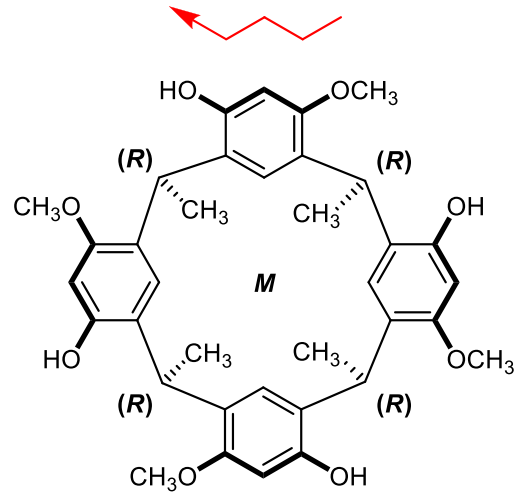

(A)

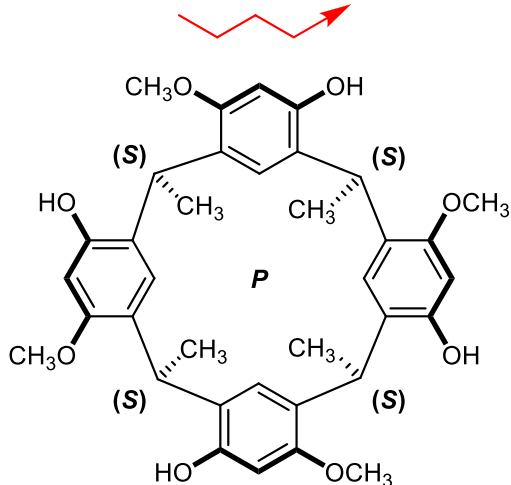

(B)

Figure 6. $C_{4}$ dissymmetric resorcinarene enantiomers with assigned stereochemistry using the CIP $R / S$ and the recently applied $M / P$ notation.

Recently,21 we have applied the $M$ and $P$ notation to describe the absolute stereogenicity of $C_{4}$ dissymmetric resorcinarenes. This notation is clearly the simplest to apply "in text" as it obviates the need for individually identifying the four 
necessarily identical stereogenic centres i.e. $R, R, R, R$ - or $S, S, S, S$-. However, this notation was intended purely as an abbreviated method for description of $C_{4}$ dissymmetric resorcinarenes and can only be applied (without complication) to $C_{4}$ dissymmetric resorcinarenes (as opposed to the $C_{1}$ or $C_{2}$ symmetric). In the event of a minor functionalisation such as that shown in scheme $\mathbf{1}$ below, the resulting $C_{1}$ symmetric derivative is better described with the usual $R / S$ notation for stereogenic centres.
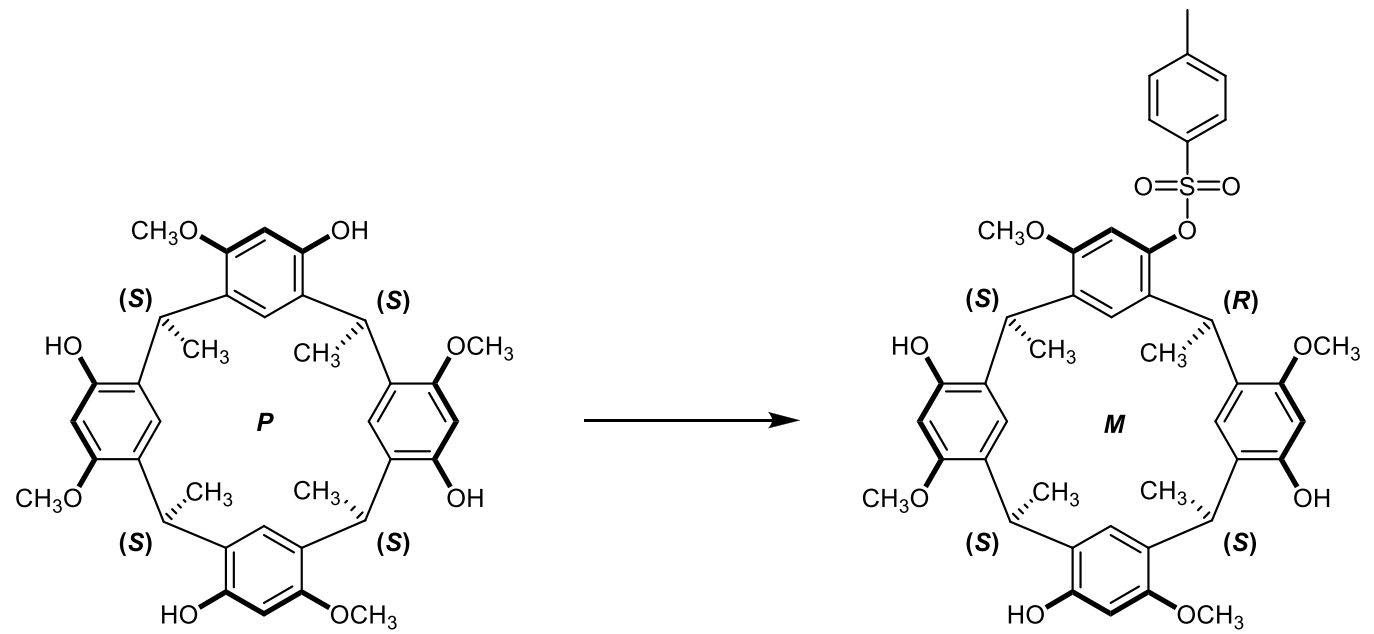

Scheme 1. Changes to the chirality sense of a resorcinarene in the event of a minor functionalisation.

We believe that in most instances preservation of the Cahn, Ingold and Prelog classification of chirality, and the methods described therein, describes the chirality of resorcinarenes very adequately. This is also true of the chiral calixarenes with special reference to the modifications recommended by Dodziuk and Mirowicz8 and the examples of Schlögl.19

\section{Structural representation of resorcinarenes and their chirality}

Many forms of resorcinarene representation have developed over the relatively brief period which they have been studied. To avoid confusion the particular depictions of resorcinarenes that will be used throughout this document are outlined here.

The most unambiguous representations of the resorcinarenes and their chirality are the three-dimensional depictions shown in figure 7. In this case the crown, chair and 
diamond conformations are shown. These are closely related to the calixarene cone, partial cone and 1,2-alternate conformations respectively.

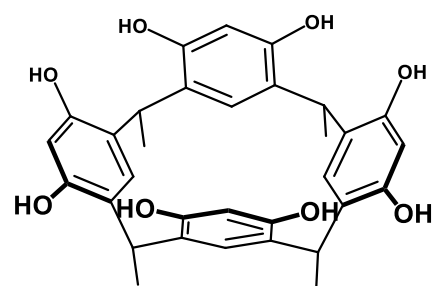

crown

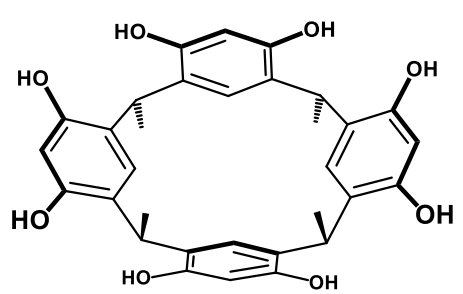

chair

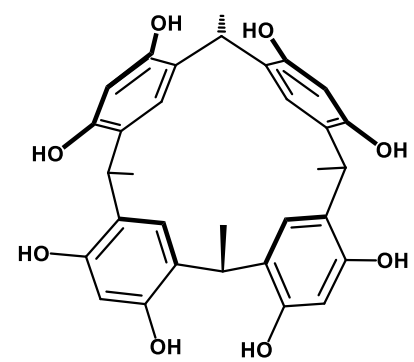

diamond

Figure 7. Three dimensional depictions of the crown, chair and diamond conformations of resorcinarenes.

However, due to the cumbersome nature of these pictures (particularly with additional functionality present) and the exceptional effort required to construct them in a clear manner, they are generally not used. Accordingly, the reader will note that where a molecule of significant complexity is being described it shall be as the "flat" type diagram shown in figure $\mathbf{8}$.

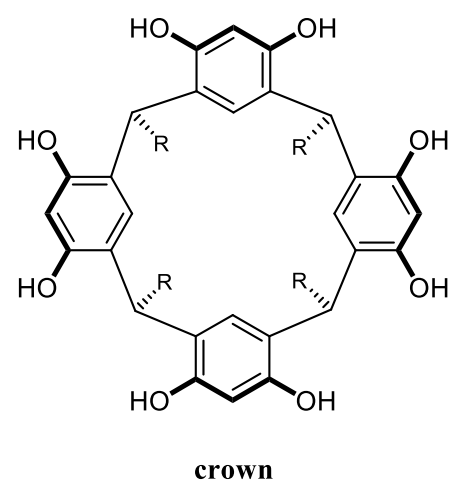

crown

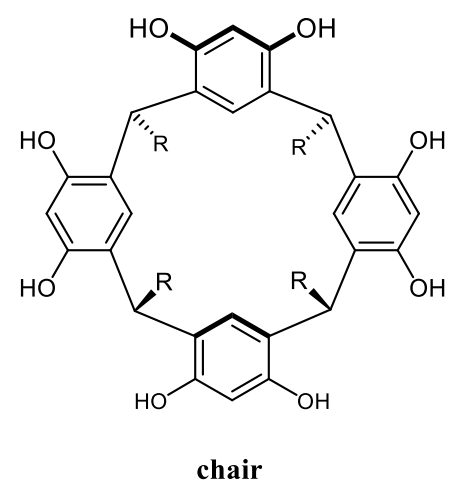

chair

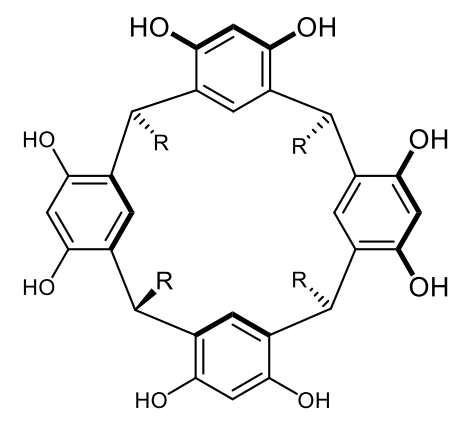

diamond

Figure 8. "Flat" style depictions of the crown, chair and diamond conformations of resorcinarenes.

In these cases the diagram will represent the resorcinarene molecule viewed from above the cavity with the orientation of the alkyl chains indicated using wedged bonds in the usual manner. 
The focus of resorcinarene research predominantly revolves around the crown isomer, as this is generally the most readily obtainable. For convenience, and for the sake of efficient usage of space, the crown isomer shall also be represented in the bracketed form shown below.<smiles>[R]C(C)(C)c1cc(C(C)(C)C)c(O)cc1O</smiles>

crown<smiles>CC(C)(C)c1cc(C(C)(C)C)c(O)c(C(C)(C)C)c1</smiles>

cone

Figure 9. "Simplified" depictions of the resorcinarene crown conformation shown with "all axial" alkyl groups and the calixarene cone conformation.

This is very similar to the commonly used depiction of the calixarene cone (also shown). However, unlike the calixarenes, the resorcinarenes possess a carbon centre with the potential for chirality. This added complication may be overcome by applying the conventions shown in figure $\mathbf{1 0 .}$

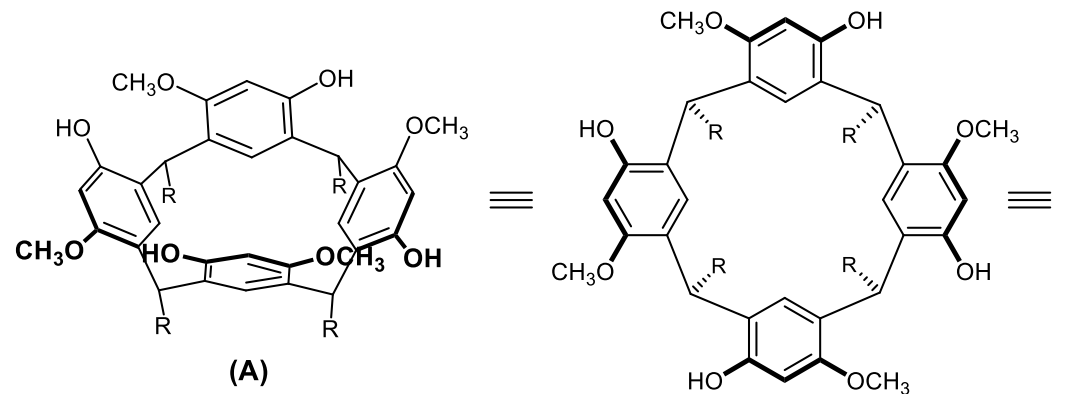

(B)<smiles>[R]C([CH])(C)c1cc(CC)c(O)cc1OC</smiles><smiles>[R]C(C)c1cc(C(C)(C)C)c(OC)cc1O</smiles>

Figure 10. Four depictions describing the absolute stereochemistry of a single chiral resorcinarene enantiomer.

The third diagram (C) is shown with the alkyl groups (R) in the all pseudo axial position and the methine proton projecting "out of the page" to indicate that the resorcinarene is being viewed from a position "outside" of the resorcinarene annulus. This depiction has the advantage that the absolute stereochemistry of the 
resorcinarene is described with a relatively efficient diagram. The resorcinarene enantiomer $(\mathbf{C})$ is described equally well by the corresponding structure (D) viewed from "inside the annulus".

The racemate of a chiral resorcinarene is perhaps best represented (when using the simplified drawing convention) with the two individual enantiomers drawn separately in the fashion shown in figure 11 (A) where both are viewed from the same perspective (from outside the annulus in this case).

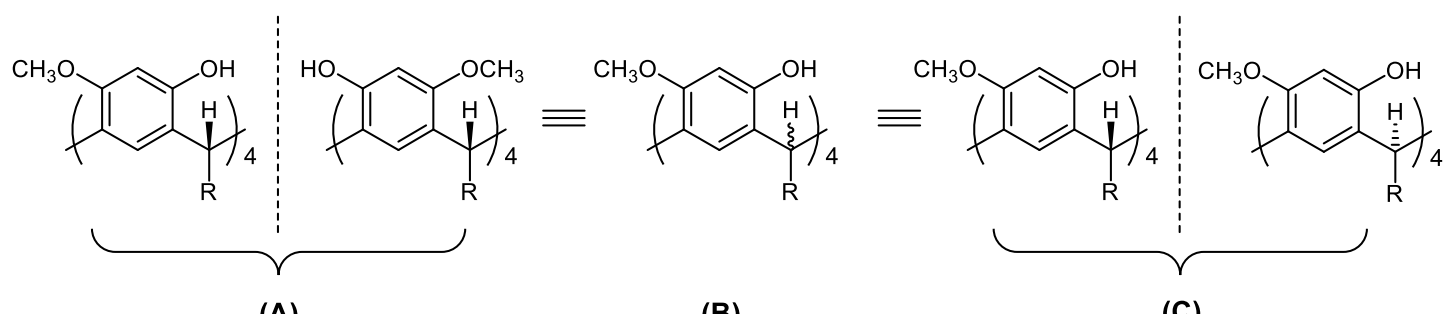

Figure 11. Simplified depictions of the resorcinarene racemate.

However, this also can become cumbersome in schematics. Where convenient, the racemate shall be drawn with a wavy bond to the methine proton (B), to distinguish it as such. This is intended to represent the two enantiomers, one being viewed from within the annulus and the other from without (C).

In 2002, IUPAC published new recommendations for the nomenclature of phanes with specific reference to the calixarene ring system6. This new system completely replaced the previous long-winded alkene cyclic alkene based nomenclature, obviating the need for a shortened independant nomenclature system for the calixarenes. It also brings calixarene numbering in to line with already well established alkane and ring system nomenclature. The IUPAC list of structural features, for which priority assignment of the lowest locant is given, is shown below:

1. numbering of phane parent hydride

2. heteroatoms introduced by skeletal replacement

3. indicated hydrogen

4. nondetachable 'hydro-'/'dehydro-' prefixes 
5. principal characteristic group (named as suffix)

6. unsaturation ('-ene'/'-yne' endings and 'hydro-'/'dehydro-' prefixes)

7. substituents named as prefixes (alphabetized substituents)

Thus the new IUPAC name for the calixarene (A) and the resorcinarene (B) below would be designated 1,3,5,7(1,3)-tetrabenzenacyclooctaphane-12,32,52,72-tetrol and 1,3,5,7(1,3)-tetrabenzenacyclooctaphane-14,16,34,36,54,56,74,76-octol. Although this name is of a adequate length to be applied in text it may be conveniently shortened by replacement of the common "1,3,5,7(1,3)-tetrabenzenacyclooctaphane" component with "calix[4]arene" to yield more familiar nomenclature shown in Figure 12.

The "correct" and "incorrect" numbering assignments of (C) are shown to exemplify the IUPAC priority rules for phane nomenclature. Specifically, the hydroxyl groups of calixarene (C), which are given the characteristic group suffix “-ol” (rule 5), precede the bromine atom (rule 7) with regard to assignment of the lowest locant. 


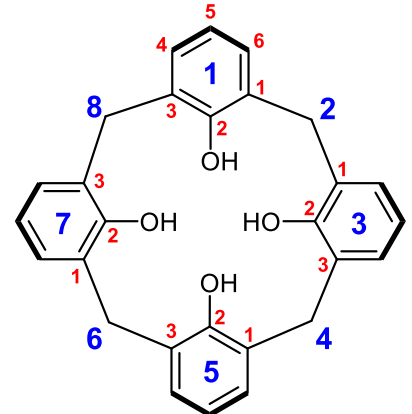

A

1,3,5,7(1,3)-tetrabenzenacyclooctaphane- $1^{2}, 3^{2}, 5^{2}, 7^{2}$-tetrol

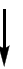

calix $[4]$ arene- $1^{2}, 3^{2}, 5^{2}, 7^{2}$-tetrol

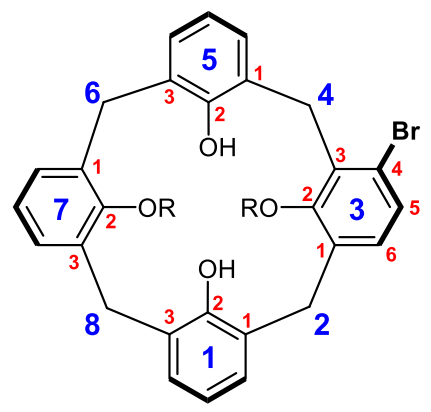

C

$3^{4}$-bromo- $3^{2}, 7^{2}$-dialkoxy-1,3,5,7(1,3)-tetrabenzenacyclooctaphane- $-1^{2}, 5^{2}$-diol $\downarrow$

$3^{4}$-bromo- $3^{2}, 7^{2}$-dialkoxycalix[4]arene- $1^{2}, 5^{2}$-diol

CORRECT

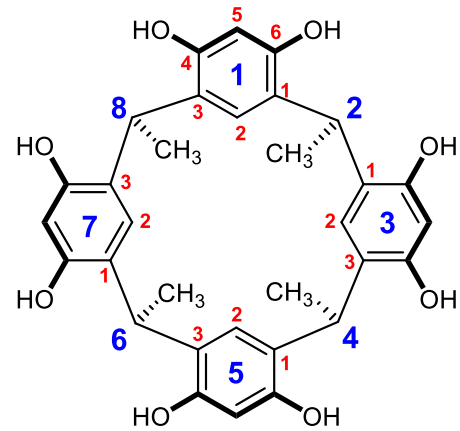

B

1,3,5,7(1,3)-tetrabenzenacyclooctaphane$1^{4}, 1^{6}, 3^{4}, 3^{6}, 5^{4}, 5^{6}, 7^{4}, 7^{6}$-octol

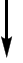

resorcin[4]arene- $1^{4}, 1^{6}, 3^{4}, 3^{6}, 5^{4}, 5^{6}, 7^{4}, 7^{6}$-octol

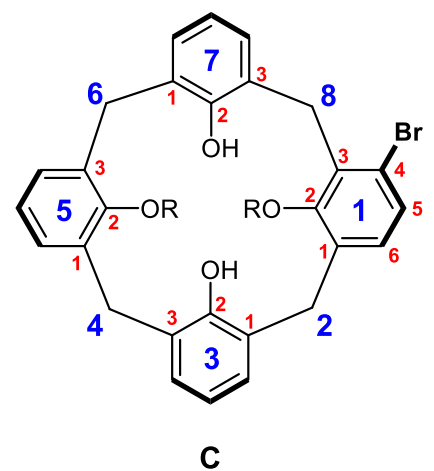

$1^{4}$-bromo-1 ${ }^{2}, 5^{2}$-dialkoxy-1,3,5,7(1,3)-tetrabenzenacyclooctaphane- $3^{2}, 7^{2}$-diol

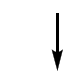

$1^{4}$-bromo- $1^{2}, 5^{2}$-dialkoxycalix[4]arene- $3^{2}, 7^{2}$-diol

INCORRECT

Figure 12. Application of the "phane" numbering to the calixarene ring system and its adaptation to the calixarene "common" name usage.

Reviews on chiral calixarenes are currently available in the literature.9,22 However, due to the many recent advances in the field, a further summation of the current work is justified.

The current review is limited to the calixarenes and resorcinarenes with helical dissymmetry of the type described in figure 3 and on which the bulk of chiral research has been focused. However, it is acknowledged that good work has also 
been reported on the minor members of the calixarene family such as the oxacalixarenes.23

The dissymmetric calixarenes and resorcinarenes with $C_{\mathrm{n}}$ symmetry do not by any means comprise the largest portion of the chiral calixarene family. However, their high symmetry 24 and simplicity makes them by far the most aesthetically pleasing. The following sections are arranged in order of increasing symmetry (i.e. $C_{1}$ to $C_{4}$ ). Although all completely asymmetric molecules must necessarily have a $C_{1}$ axis of symmetry, since the axis about which the calix defines is preserved in all examples described herein, it is deemed logical to begin by examining those molecules which have one moiety positioned about that axis affording a "direction" in the spirit of helix-like dissymmetry. This should then sensibly be followed by two groups $\left(C_{2}\right.$ symmetric) and finally four groups ( $C_{4}$ symmetric). The consequence of such a structural arrangement is the necessary repetition of references to publications containing molecules from more than one symmetry group i.e. a publication containing a calixarene with $C_{2}$ symmetry and another having $C_{4}$ symmetry must necessarily be referenced in both sections.

\section{The $C_{1}$ dissymmetric}

The chiral calixarenes and resorcinarenes having $C_{1}$ symmetry are perhaps the least "attractive" of the family in terms of aesthetics. However, they are significantly appealing with regards to the simplicity of their preparation from the parent macrocycles.

\section{Calixarene based}

Of the methods used to prepare chiral derivatives from the parent macrocycles the most flexible approach appears to be via the calix[4]quinones. The monoquinone $\mathbf{2}$ is prepared by direct oxidation of the triether 1 with thallium(III) triflate in good yield (51\%25). Compound 2 can then be readily converted into a variety of racemic 
dissymmetric derivatives by 1,4-addition with generally greater than $60 \%$ conversion (Scheme 2).

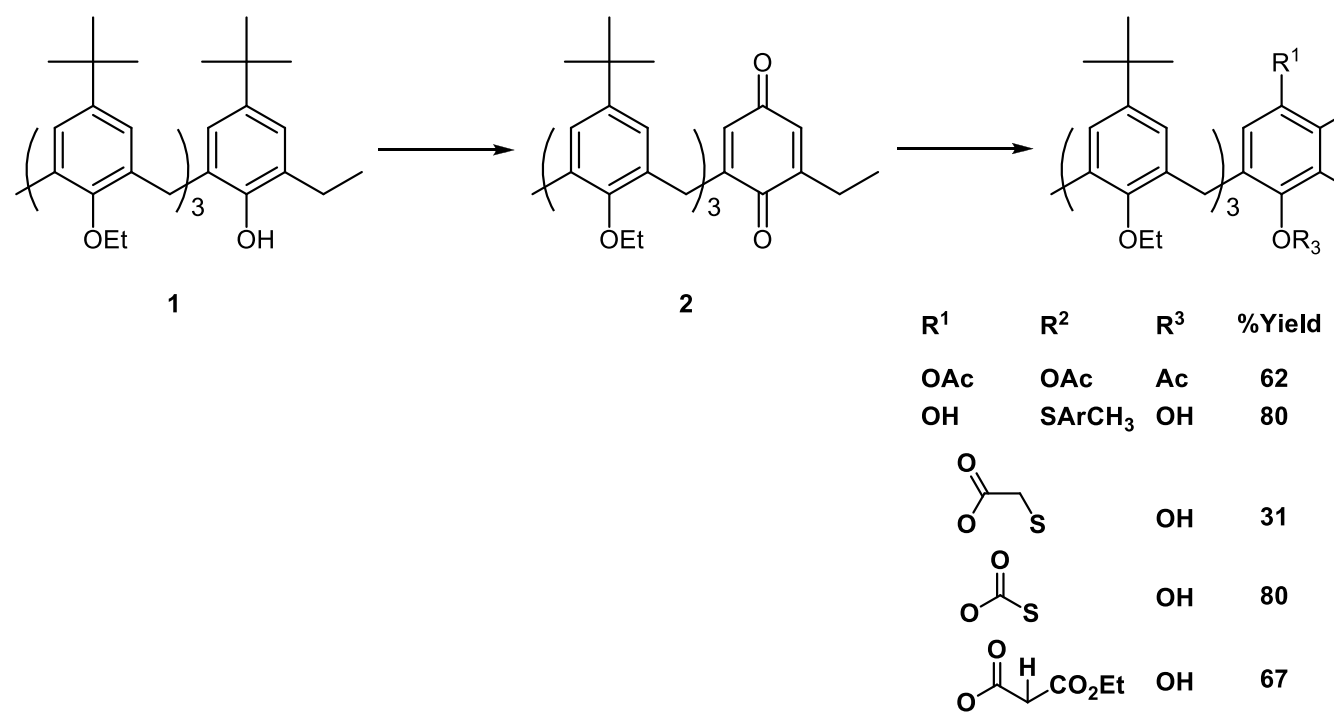

Scheme 2. The preparation of chiral calixarenes from mono-quinone 2.

Gutsche demonstrated the chirality of the prepared materials by n.m.r. spectroscopy in the presence of Pirkle's reagent $((S)-(+)-2,2,2$-trifluoro-1-(9-anthryl)ethanol).25 All of the compounds showed doubling of peaks however no efforts appear to have been made to resolve the racemic mixtures.

Similar materials have been prepared during the oxidation of the tripropyl ether 3 to the monoquinone 4.26 The meta substituted iodide 5 was obtained in $32 \%$ yield in addition to the monoquinone which was obtained in $63 \%$ yield. The iodide 5 was then reduced to the hydroquinone $\mathbf{6}$ with sodium borohydride. 


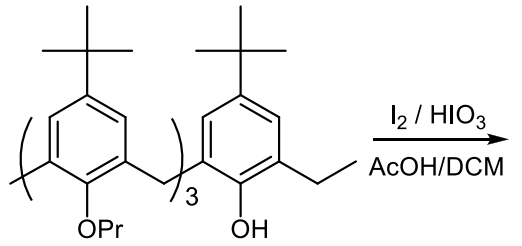

3<smiles>CCC1=CC(=O)C=C(CC)C1=O</smiles>

$464 \%$

5

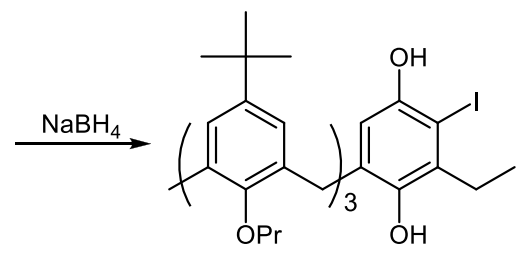

6

Scheme 3. Iodination of the calixarene triether $\mathbf{3}$ to afford the asymmetric monoiodide $\mathbf{5}$ and its subsequent reduction to $\mathbf{6}$.

The n.m.r. spectroscopic analysis of $\mathbf{5}$ and $\mathbf{6}$ appear indicative of mono-meta substituted species but no further proof of chirality was offered.

Verboom and coworkers have also introduced a meta group via the slightly more flexible approach of electrophilic substitution adjacent to the acetamide functionality of compounds 7 and 827 (scheme 4). This method allowed the preparation of 9 and $\mathbf{1 0}$ by direct nitration in 91 and $98 \%$ yield respectively. Bromination of $\mathbf{7}$ and $\mathbf{8}$ afforded their respective monobromides (11 and 12) in 64 and $58 \%$. Compounds 9 12 were demonstrated to be racemic by $1 \mathrm{H}$ n.m.r. spectroscopy in the presence of Pirkle's reagent, however, none of the racemates were resolved.

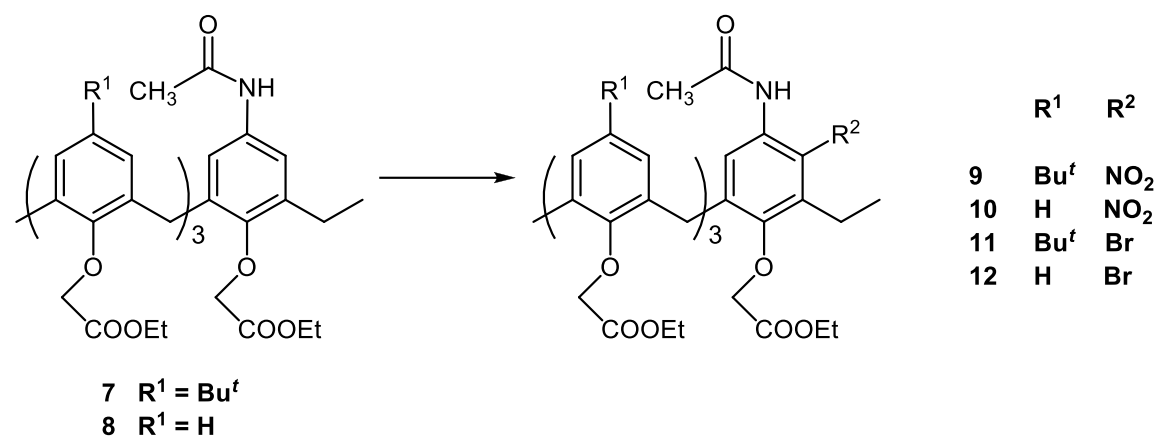

Scheme 4. Bromination or nitration of calixarenes $\mathbf{7}$ and $\mathbf{8}$ to afford the corresponding chiral monobromides and nitrates. 
Huang et al.17 made the logical advancement to Verboom's method with the introduction of an N-Boc-L-proline amide to afford calixarene 13. Bromination or nitration of the ring positions adjacent to the amide resulted in the direct formation of diastereomers of $\mathbf{1 4}$ and $\mathbf{1 5}$ in good yields (Scheme 5).

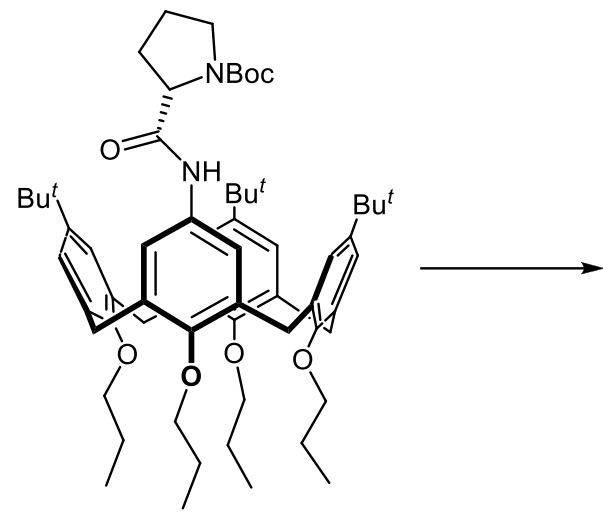

13

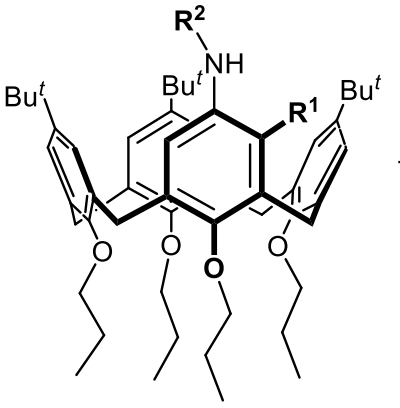

a

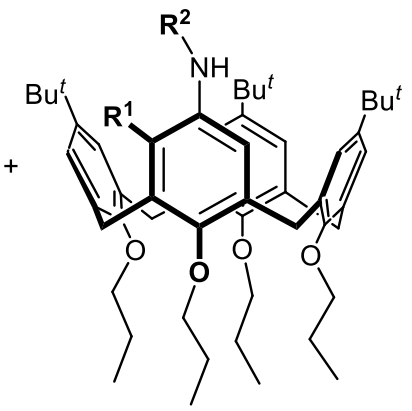

b

$\begin{array}{lll} & \mathrm{R}^{1} & \mathrm{R}^{2} \\ 14 & \mathrm{Br} & \text { N-Boc-L-Pro } \\ 15 & \mathrm{NO}_{2} & \text { N-Boc-L-Pro } \\ 16 & \mathrm{NH}_{2} & \text { N-Boc-L-Pro } \\ 17 & \mathrm{~N}\left(\mathrm{CH}_{3}\right)_{2} & \text { N-Boc-L-Pro } \\ 18 & \mathrm{Br} & \mathrm{H} \\ 19 & \mathrm{NO}_{2} & \text { H } \\ 20 & \mathrm{~N}\left(\mathrm{CH}_{3}\right)_{2} & \text { L-Pro }\end{array}$

Scheme 5. Functionalisation of the chiral amide $\mathbf{1 3}$ to afford diastereomeric species.

The diastereomers were resolved by preparative t.l.c. or column chromatography. Removal of the $N$-Boc-L-proline auxiliary was achieved by hydrolysis with

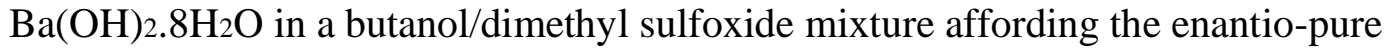
primary amines of $\mathbf{1 8}$ and $\mathbf{1 9}$. The laevorotary enantiomer $18 \mathbf{b}([\alpha] 25 \mathrm{D}-15.9, \mathrm{c}=1$ (DCM)) was determined by $\mathrm{X}$-ray crystallography to have the $P$ configuration, as shown in scheme 5. The methodology was extended further 15 by reduction of the nitro diastereomers 15 with hydrazine to give the anilines $\mathbf{1 6}$ which were methylated to yield the diastereomeric tertiary amines 17. Removal of the Boc protecting groups with trifluoroacetic acid in dichloromethane gave the diamines 20 a and 20 b which were examined as organocatalysts for the enantioselective aldol reaction. 15

Perhaps a simpler example is the restricted calix[6] arene derivative 21 (Figure 13) produced by Shinkai.28 The 4-methoxy- $m$-xylenyl moiety bridges the 1,5-aryl units 
and is restrictive enough to prevent ring inversion and therefore racemisation. The chirality of $\mathbf{2 1}$ was proven by n.m.r. spectroscopy with Pirkle's reagent, and subsequent resolution by enantioselective HPLC (Daicel Chiralpak AD, $n$-hexane/2propanol 98:2), though the absolute stereochemistry was not determined.

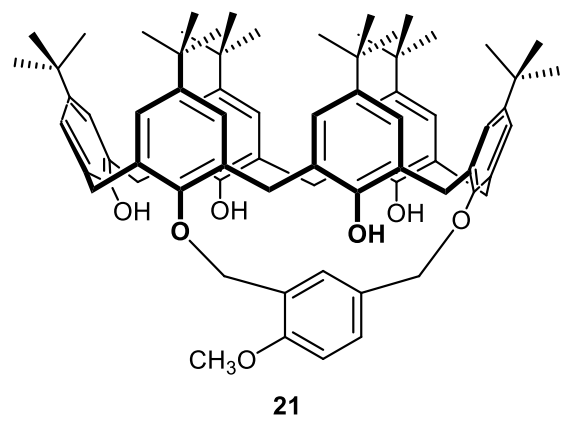

Figure 13. One enantiomer of a chiral 1,5- linked hexamer produced by Shinkai.28

Fragment condensation for the preparation of $C$ dissymmetric calixarenes is generally a much more cumbersome and low yielding approach. However, stepwise synthesis is a proven method for the preparation of the achiral calixarenes 29 and planar chiral calixarenes.12,30 Consequently, it has also found its place in the generation of chiral species.

Casabianca and coworkers 31 performed several $3+1$ condensations using the bisbromomethyl phenols with $\mathrm{TiCl}_{4}$ in dioxane to produce the chiral calixarenes 22 25 given in scheme 6. These were afforded in yields of 10-35\%. A single crystal Xray structural determination of $\mathbf{2 5}$ was reported later.32<smiles>[R]c1ccc(O)c(Cc2cc([R])ccc2Cc2cc([R])c([R])c([R])c2O)c1</smiles>

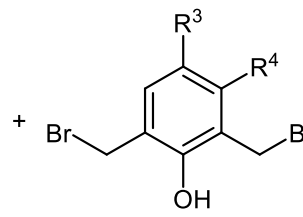<smiles></smiles>

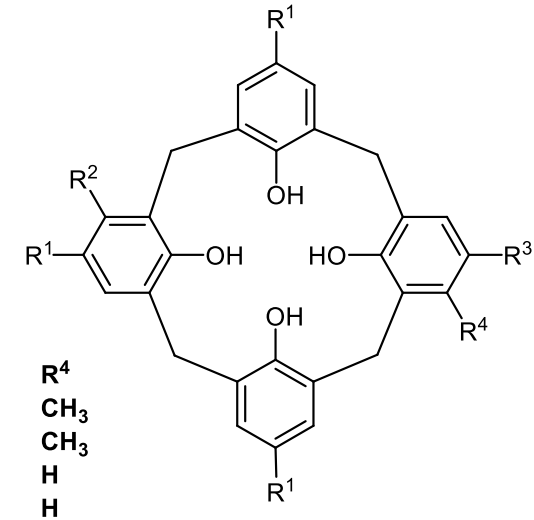

$$
\begin{array}{llll}
\mathrm{R}^{1} & \mathrm{R}^{2} & \mathrm{R}^{3} \\
\mathrm{CH}_{3} & \mathrm{H} & \mathrm{CH}_{3} \\
\mathrm{Pr}^{i} & \mathrm{H} & \mathrm{Pr}^{i} \\
\mathrm{Pr}^{i} & \mathrm{CH}_{3} & \mathrm{Ph} \\
\mathrm{Pr}^{i} & \mathrm{CH}_{3} & \mathrm{Pr}^{i}
\end{array}
$$$$
\mathrm{CH}_{3} \mathrm{H} \quad \mathrm{COC}_{6} \mathrm{H}_{5} \mathrm{OH}
$$ 
Scheme 6. Some 3+1 fragment condensation reactions to produce dissymmetric calixarenes having $C_{1}$ symmetry.

In a similar 3+1 condensation Böhmer has incorporated a resorcinol unit linked via its 2,6- positions 33 to give compound 26 (scheme 6). In this case the chirality was demonstrated at low temperatures $\left(-40^{\circ} \mathrm{C}\right)$ by n.m.r. spectroscopy with Pirkle's reagent. At such low temperatures the ring inversion is slow on the n.m.r. time scale and interaction of the enantiomers with the reagent may be observed.

Shinkai went one step further and locked the chiral calixarene into the cone conformation via a selective propylation.34 The calixarene 27 (scheme 7), prepared using the standard 3+1 fragment condensation method in $9 \%$ yield, was initially propylated with barium hydroxide as the base to give the tri-ether $\mathbf{2 8}$ and then converted to the tetra-ether 29 using standard conditions ( $\mathrm{PrBr}, \mathrm{NaH}, \mathrm{DMF} / \mathrm{THF}$ ). This stepwise alkylation procedure avoided the complication of multiple confomational isomers produced by direct per-alkylation of calixarene 27 . The chirality of 28 was demonstrated by n.m.r. spectroscopy at low temperatures $\left(-40^{\circ} \mathrm{C}\right)$ in the presence of Pirkle's reagent. More significantly, the tetra-ether 29 was resolved to its enantiomers using enantioselective HPLC (Daicel ChiralPak OP(+), hexane/2-propanol/methanol 1:3:16).

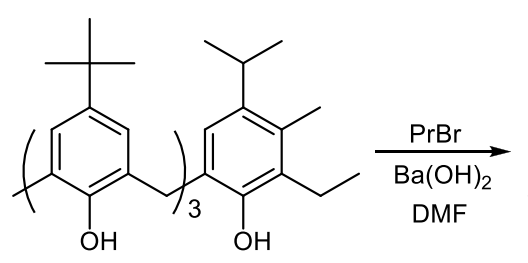

27

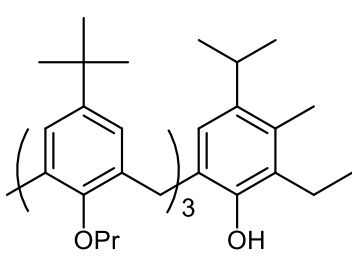

28

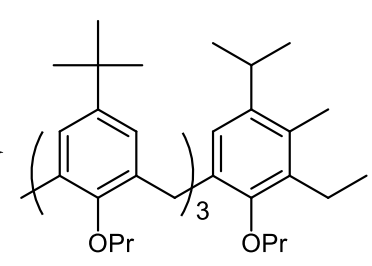

29

Scheme 7. Sequential alkylation at the lower rim of calixarene 27 to prevent racemisation.

The reverse approach was also adopted by Böhmer.35 By preparing the mono or triether derivatives of the $C_{4}$ dissymmetric calixarene $\mathbf{3 0}$ (Figure 14) the 'lower' symmetry materials $\mathbf{3 1}$ - $\mathbf{3 5}$ were obtained. 


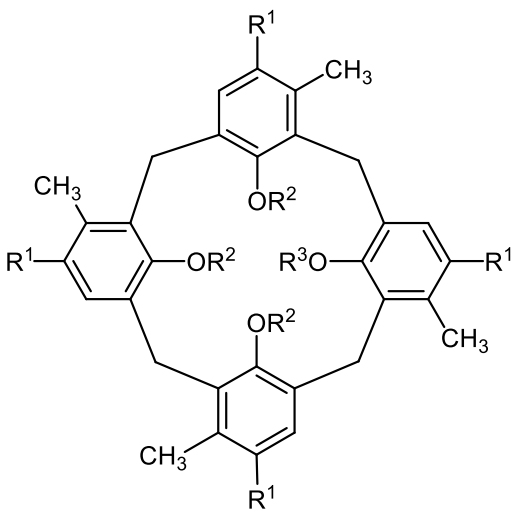

$\begin{array}{llll} & \mathbf{R}^{1} & \mathbf{R}^{2} & \mathbf{R}^{3} \\ 30 & & & \\ 31 & \mathrm{CH}_{3} & \mathrm{H} & \mathrm{H} \\ 32 & \mathrm{CH}_{3} & \mathrm{H} & n \mathrm{Bu} \\ 33 & \mathrm{CH}_{3} & \mathrm{H} & \mathrm{CH}_{2} \mathrm{C}_{6} \mathrm{H}_{4} \mathrm{NO}_{2} \\ 34 & \mathrm{CH}_{3} & \mathrm{H} & \mathrm{CH}_{3} \\ 35 & \mathrm{Pr}^{i} & \mathrm{CH}_{2}{ }^{i} \text { COOEt } \mathrm{H}\end{array}$

Figure 14 - Desymmetrisation of $C_{4}$ dissymmetric calixarene 30 by alkylation at the lower rim.

Shinkai36 developed a stepwise process to construct a naphthalene unit on the calixarene (scheme 8). The product (36) is locked in the cone conformation and so cannot racemise. The chirality was established by n.m.r. spectroscopy in the presence of Pirkle's reagent, however, it was not resolved.

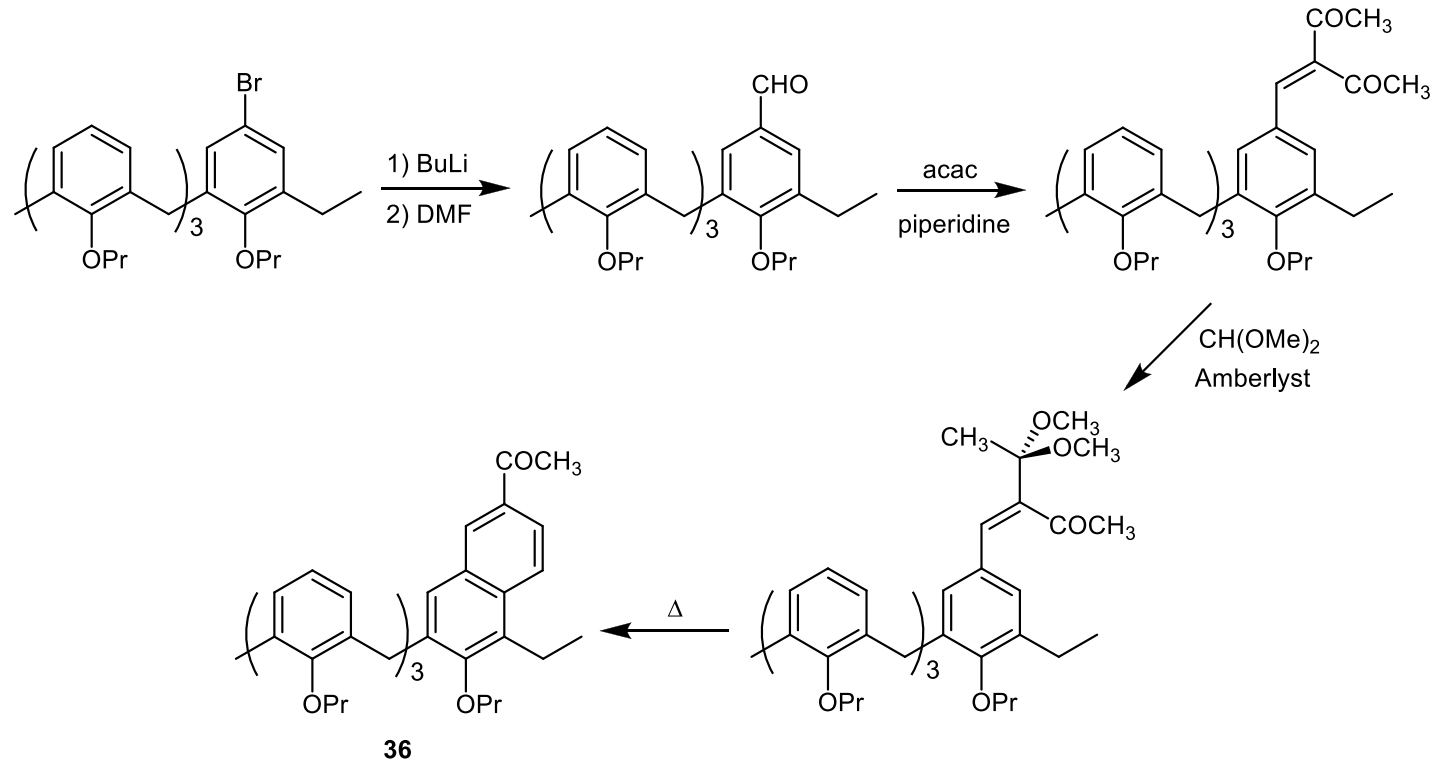

Scheme 8. Scheme for the preparation of the $C_{1}$ symmetric naphthalene derivative 36 (note: acac $=2,4$-pentanedione). 
More recently a novel approach was adopted by Biali.37 Treatment of the bis(spirodienol) 37 with diethylamino sulfur trifluoride (DAST) afforded the monofluoro compound $\mathbf{3 8}$ in $8 \%$ yield. However, this compound is not locked into a particular conformation and can therefore readily racemise.

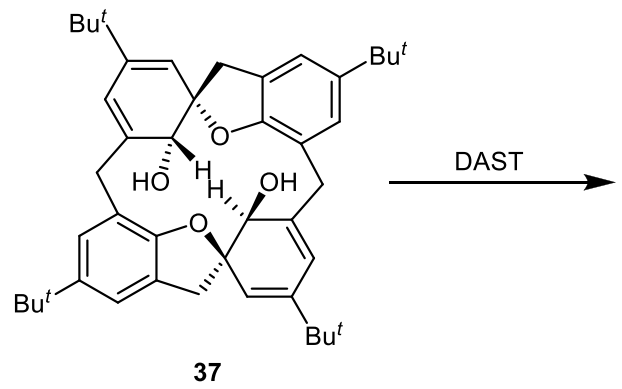

37

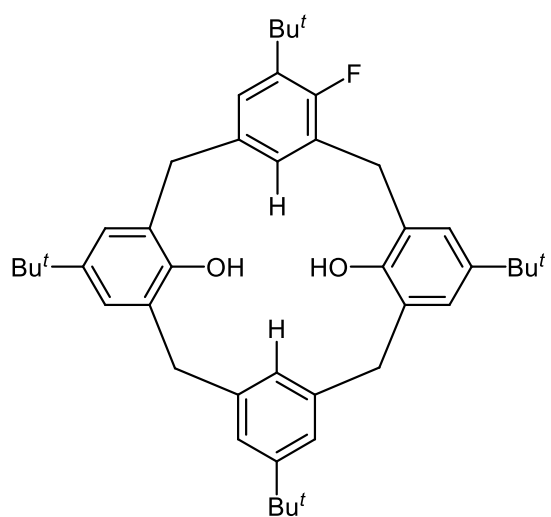

38

Scheme 9. Fluorination of compound $\mathbf{3 7}$ affords the asymmetric monofluoride $\mathbf{3 8}$.

\section{Resorcinarene based}

The preparation of resorcinarene derivatives having $C_{1}$ dissymmetry is significantly simpler than the oxidation and fragment condensations of the calixarenes. Yet surprisingly there are fewer examples. Asymmetry may be introduced simply by alkylation of one of the parent resorcinarene phenols 38 (scheme 10) as is the case for the p-methylbenzyl ether 39 (32\%). Per-acetylation to give 40 in $64 \%$ yield and subsequent cleavage of the benzylic ether yielded the heptaacetate 41 (23\%). The resorcinarene derivatives were not resolved, however, n.m.r. spectroscopic studies of 39 in the presence of $(R)-N, N, N$-trimethyl- $\alpha$-methylbenzylammonium iodide showed doubling of the resorcinarene signals. 


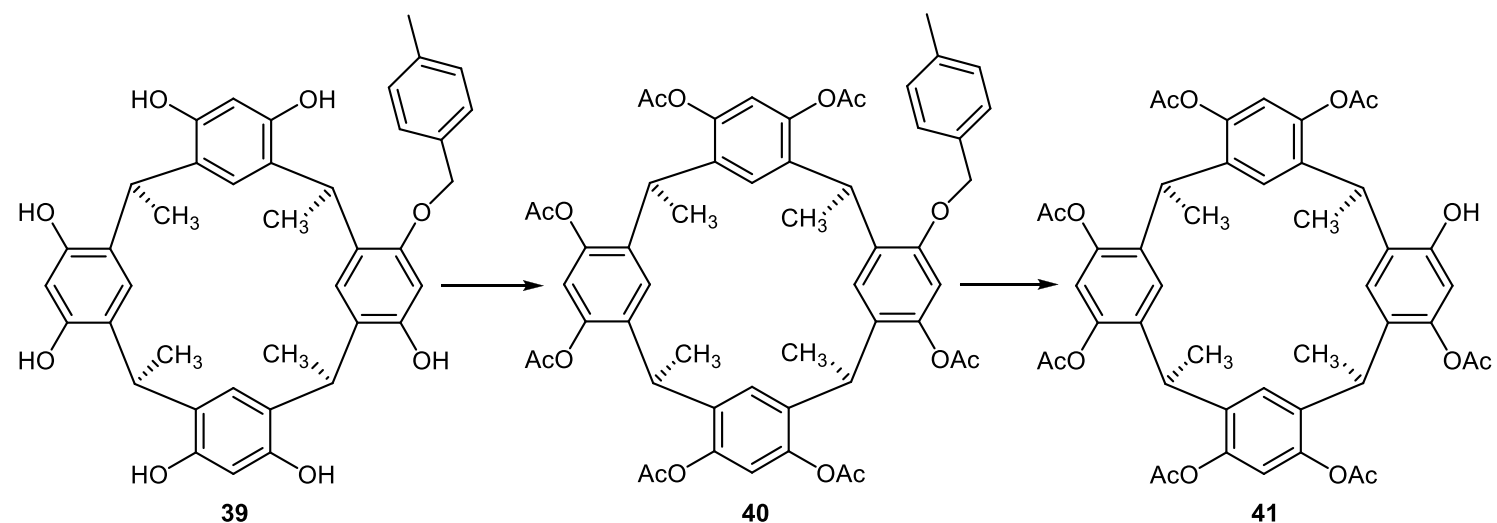

Scheme 10. Introduction of asymmetry to $C$-methylcalix[4]resorcinarene by monobenzylation (to afford 39) and subsequent transformations.

Mattay and coworkers 39 took the more efficient approach of generating dissymmetry with the introduction of a single chiral moiety. The reaction of camphorsulfonyl chloride with the parent resorcinarene (scheme 11) produces the diastereomers 42 and 43, making the resolution of enantiomers unnecessary. The diastereomers were separated by normal phase HPLC and methylated to give 44. The chiral auxiliary was then removed to afford the heptamethyl ether enantiomer $\mathbf{4 5}$. 


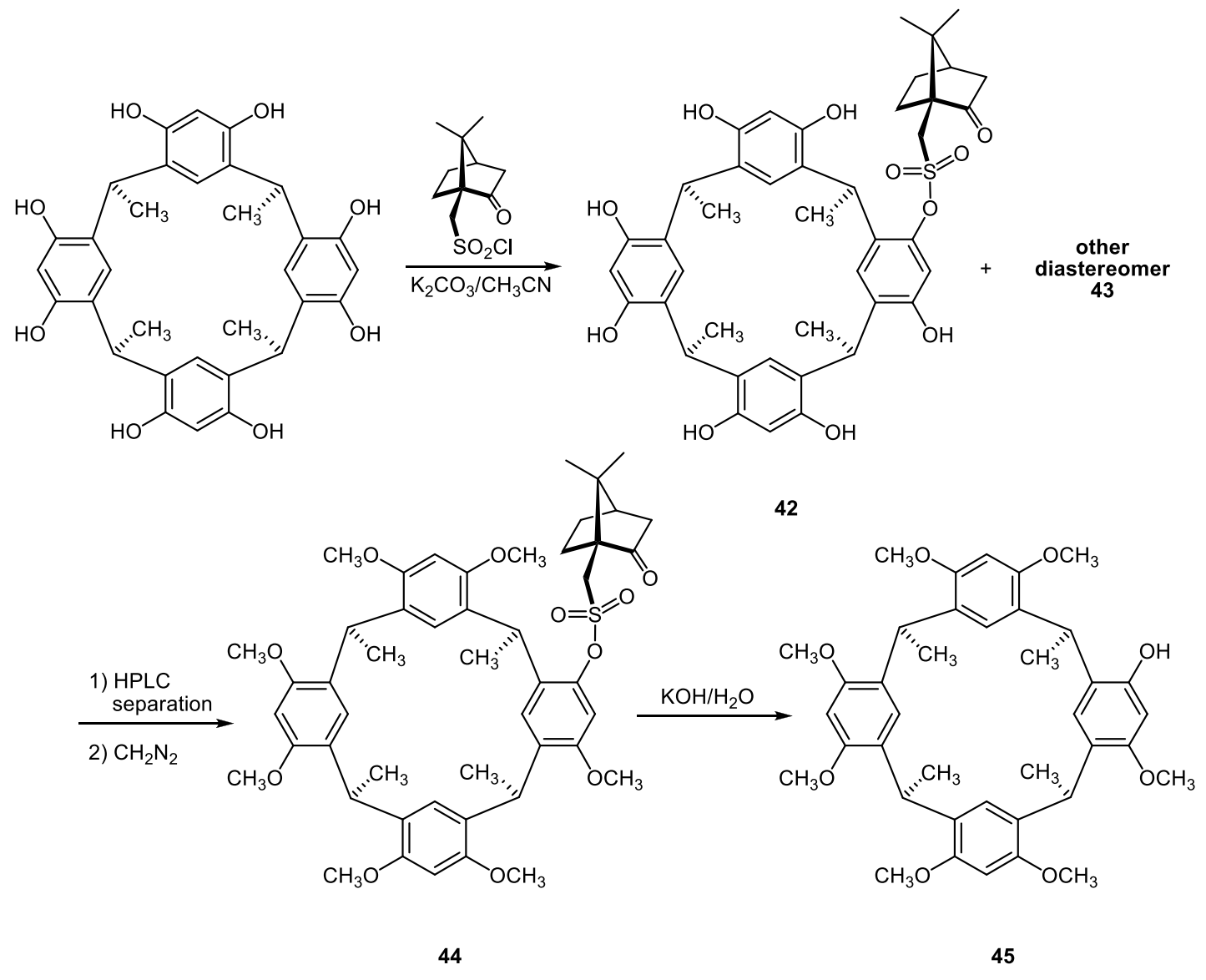

Scheme 11. Preparation of the enantiomers of the heptamethylether of $C$ methylcalix[4]resorcinarene (45). 


\section{The $C_{2}$ dissymmetric}

\section{Calixarene based}

Few examples of fragment condensations to produce meta substituted $C_{2}$ dissymmetric materials exist. The only example of note is that described by Böhmer40 (scheme 12). Condensation of the hydroxymethylated bisphenol 46 with titanium tetrachloride gave the calixarene $\mathbf{4 7}$ in $18 \%$ yield. Böhmer attributes the lack of interest in compounds of this type to the substantial efforts required to prepare the bisphenol $\mathbf{4 6}$ which requires five separate reaction steps. The desired cyclised material $\mathbf{4 7}$ is obtained in an overall yield of only $3 \%$ as a result.<smiles>Cc1cc(CO)c(O)c(Cc2c(O)ccc(C)c2C)c1</smiles>

46<smiles>CCCCCc1c(C)cc(CCCC)c(O)c1Cc1c(C)c(C)cc(C)c1O</smiles>

47

Scheme 12. The $2+2$ fragment condensation of benzylic alcohol 46 to give the corresponding $C_{2}$ dissymmetric calixarene 47.

Alternatively, dialkylation of the more readily obtained $C_{4}$ dissymmetric calixarenes 35 produces the lower symmetry diethers $\mathbf{4 8}-\mathbf{5 5}$ shown in figure $\mathbf{1 5}$.
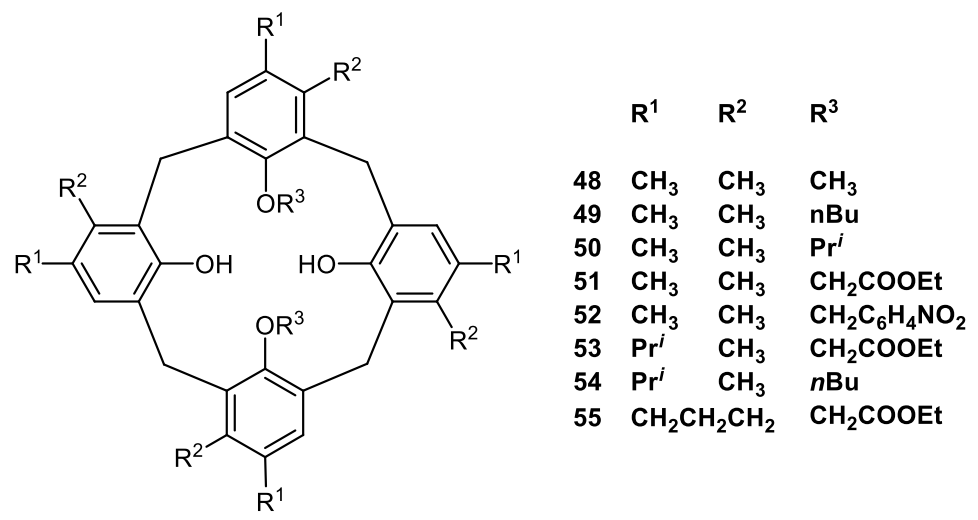

Figure 15. $C_{2}$ dissymmetric calixarenes produced by 1,5-distal dialkylation of $C_{4}$ dissymmetric calixarenes. 
Compounds 48, 51, 53, and 55 were later resolved by Böhmer41 by HPLC on a ChiralPak AD column. The decamethyl derivative $\mathbf{4 8}$ is not fixed in the cone conformation and the energy barrier for interconversion of the enantiomers was determined by examining their CD spectra as a function of time.

Another route to the meta-substituted $C_{2}$ dissymmetric calixarenes is via the quinone route developed by Gutsche.25 The calixarene-1,5-diquinones undergo 1,4nucleophilic additions in a similar manner to the monoquinones. However, the reaction is complicated by the concomitant formation of the $C_{2 \mathrm{v}}$ isomer (57). Consequently the desired isomer $\mathbf{5 6}$ is recovered in only $14 \%$ yield.

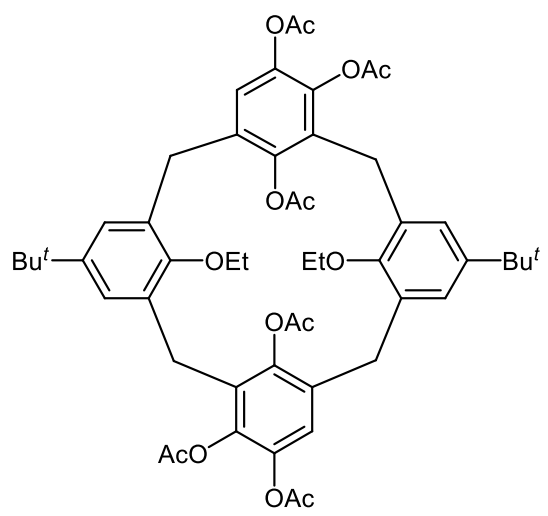

56

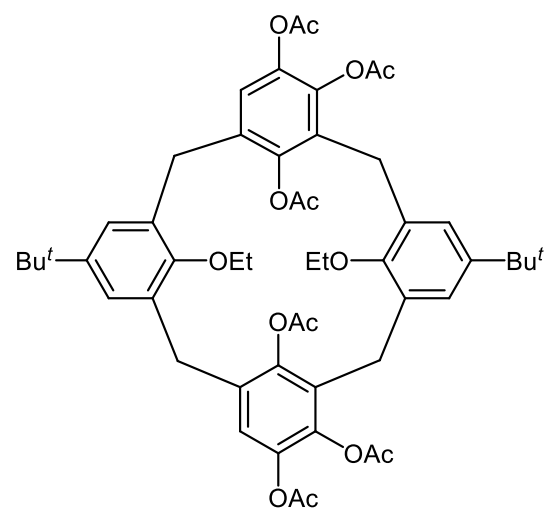

57

Figure 16. Regio-isomers 56 and 57 produced by 1,4-addition to a calixarene-1,5diquinone.

A regioisomeric mixture was also obtained 27 by bromination of the bisacetamide $\mathbf{5 8}$ to give a $10 \%$ recovery of the dibromide 59. Nitration was substantially more selective yielding $53 \%$ of the desired isomer $\mathbf{6 0}$. Significantly these calixarenes were prepared as the tetrapropyl ethers and thus are conformationally fixed and so can not racemise by ring inversion. The structure of $\mathbf{5 9}$ (as the racemate) was proven by single crystal X-ray determination, however the racemates were not resolved. 


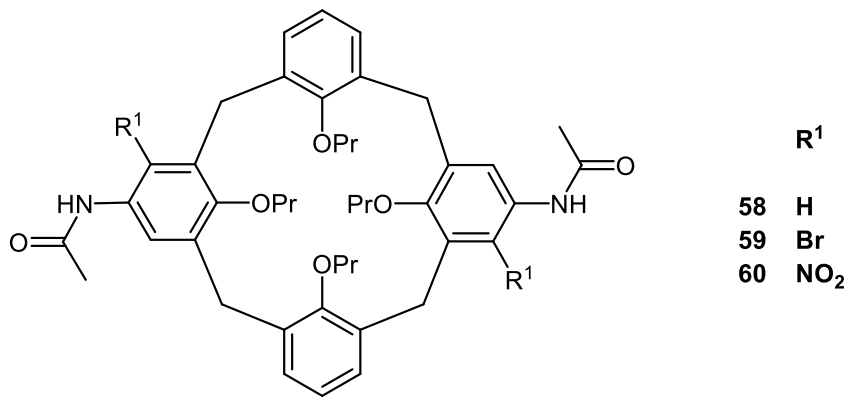

Figure 17. Chiral dibromo- and dinitro- derivatives produced by bromination and nitration of the bis-acetamide $\mathbf{5 8}$ respectively.

The propensity of the calixarenes for 1,5- distal functionalisation on both the upper and lower rims 42 lends them to the preparation of a variety of $C_{2}$ dissymmetric materials. The calixarene 1,5-diester $\mathbf{6 1}$ was effectively exploited by Bitter43 for the preparation of proximal doubly bridged calix[4]arenes such as $\mathbf{6 2}$ shown in figure 18.<smiles>[R1]c1cc(Cc2cc([R1])cc(C(C)C)c2OCC(=O)OCC)c(O)c(CC([Z20])(C)C)c1</smiles>

61

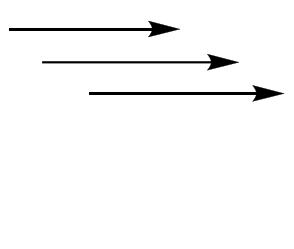

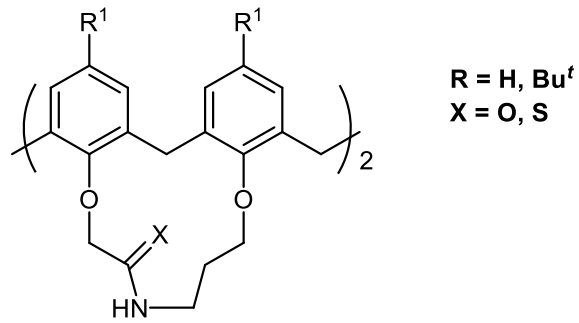

62

Figure 18. Linking of adjacent phenolic groups to generate dissymmetric calixarene 62.

The chirality of the amides and their sulfur analogues was investigated by n.m.r. spectroscopy with Pirkle's reagent to give characteristic doubling of signals.

However, resolution by enantioselective HPLC had been unsuccessful up to the point of publication.

Also of interest in this regard are the 1,5-bridged compounds produced by Böhmer 6335 and also $\mathrm{Wu}$ 64.44 While the latter example may be considered chirality by attachment, the product does have fixed $C_{2}$ symmetry. The former example is chiral 
by virtue of the conformational rigidity of the amide bridge. This was clearly demonstrated by the doubling of the n.m.r. signals in the presence of Pirkle's reagent.

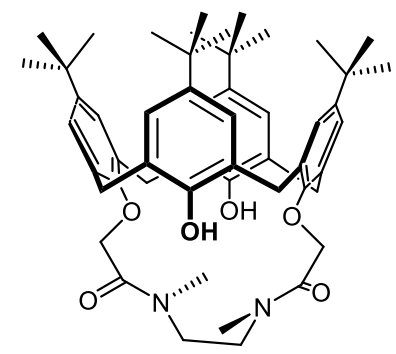

63

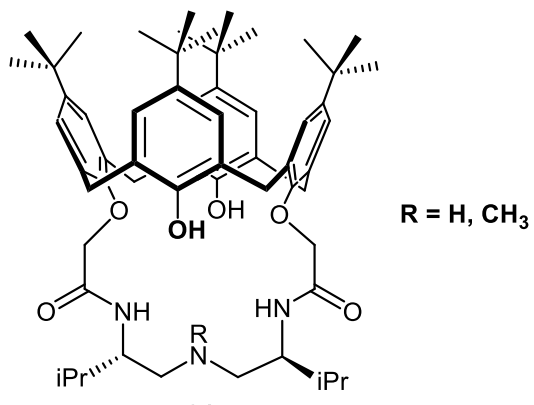

64

Figure 19. Chiral $C_{2}$ dissymmetric derivatives produced by linking distal phenolic groups.

Of the upper rim examples, the compounds prepared by Shinkai36,45 from the tetrachloromethylated calixarenes are a noteworthy example. The proximally bridged salicylic acid derivative $\mathbf{6 5}$ was produced in $36 \%$ yield directly from tetrachloromethylcalix[4]arene. Similarly the hydroxymethyl-2-naphthol bridged compound 66 was prepared in low yield. The latter was resolved by enantioselective HPLC (Daicel Chiralpak AD).<smiles>CCCOc1ccccc1Cc1cc(COc2ccccc2C(=O)OCc2cc(CC(C)C)c(OCCC)c(CC(C)(C)C)c2)cc(CC(C)(C)C)c1OCC</smiles>

65<smiles>[Z]C(C)(C)Cc1cc(COCc2cc3ccccc3cc2OCc2cc(CC(C)C)c(OCC)c(CC(C)C)c2)cc(CC(C)C)c1OCC</smiles>

66

Figure 20. Proximal bridging at the upper rim to produce dissymmetry. 


\section{Resorcinarene based}

The largest contribution to the $C_{2}$ dissymmetric family are the 1,5-bisbenzoxazines prepared by Böhmer.46 A number of derivatives were prepared from the distal tetratosylates including several bridged bisbenzoxazines (Figure 21).
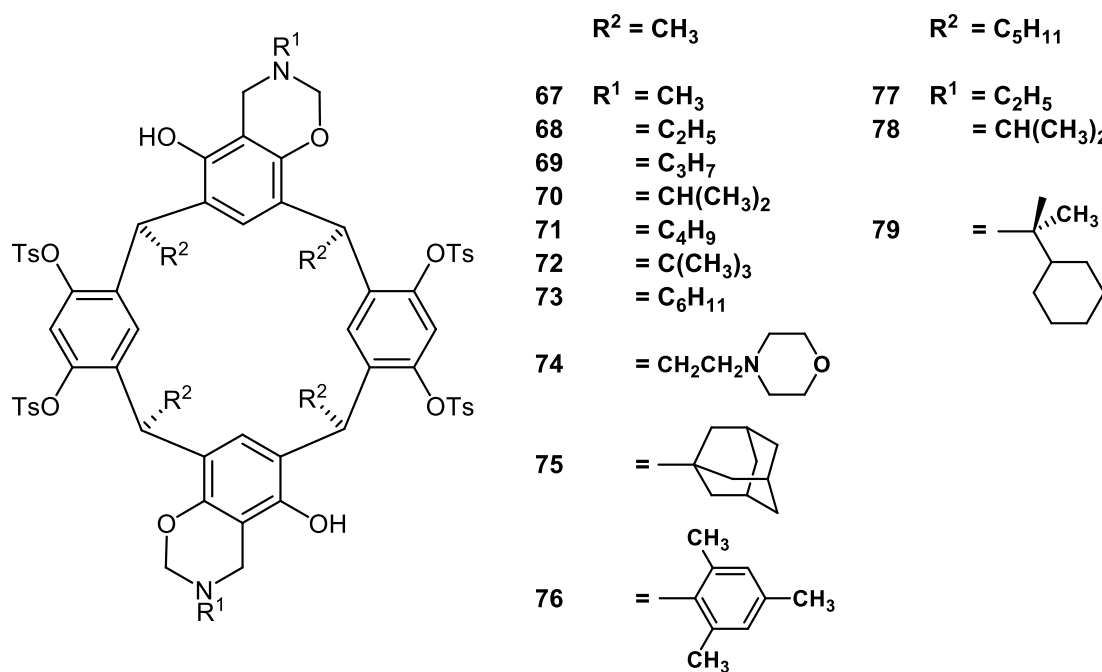

Figure 21. $C_{2}$ dissymmetric resorcinarene bisbenzoxazine derivatives produced from two distal tetra-tosylates.

More recently, the racemic bis-crowns $\mathbf{8 1}$ and $\mathbf{8 2}$ have been produced by Salorinne and Nissinen.47 Desymmetrisation of the tetramethoxyresorcinarene $\mathbf{8 0}$ with tri- and tetraethylene glycol afforded the $C_{2}$ symmetric derivatives in 18 and $22 \%$ yields respectively.<smiles>CCc1cc(C(C)C)c(O)cc1OC</smiles>

80
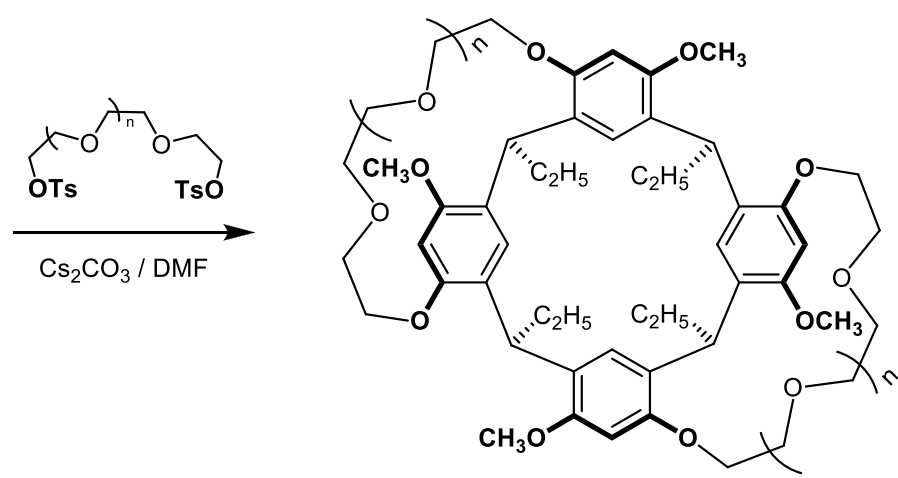

$81 n=1$

$82 n=2$

Scheme 13. Formation of the racemic resorcinarene bis-crowns from the tetramethoxy resorcinarene $\mathbf{8 0}$. 


\section{The $C_{4}$ dissymmetric}

\section{Calixarene based}

No example of a $C_{4}$ dissymmetric calixarene appears to have been generated from the parent calixarenes. This is no doubt due to the fact that introduction of a meta substituent would be significantly complicated by the formation of regioisomers. An extenuating factor in this regard would likely be that the preparation of $C_{4}$ dissymmetric calix[4]arenes by fragment condensation is substantially easier and higher yielding than the corresponding $C_{1}$ or $C_{2}$ dissymmetric derivatives. This is a direct result of the ease of preparation of the starting benzylic alcohol.

Consequently, many excellent examples of condensations of benzyl alcohols to give $C_{4}$ dissymmetric calixarenes have been published.

The earliest preparation of this kind was provided by Wu.48 The condensation of 2,6dimethyl-4-methoxybenzyl alcohol in the presence of aluminium chloride afforded the cyclic tetramer $\mathbf{8 3}$ with four 'exo' methoxy groups in $80 \%$ yield.<smiles>COc1cc(C)c(CO)c(C)c1</smiles><smiles>CCCc1c(C)cc(OC)c(C)c1CC</smiles>

83

Scheme 14. Condensation of 2,6-dimethyl-4-methoxybenzyl alcohol to afford the chiral calixarene 83 .

The first of the standard calix[4]arene examples was produced by Böhmer.35,40Benzyl alcohols 84 and 85 were treated with titanium tetrachloride in dioxane to give their respective tetramers (86 and 87). Alkylation of the phenols gave the tetra-ester cone conformers 88 and $\mathbf{8 9}$ in 62 and $34 \%$ respectively. Swager et al.49 resolved the octamethyl compound (86) as the diastereomeric tungsten(VI) adducts (90), prepared with two different chiral diols, by HPLC. The hydrobenzoin derivative (90) was also separable by flash chromatography. 
<smiles>Cc1cc(O)c(CO)cc1Br</smiles>

$84 \mathrm{R}^{1}=\mathrm{CH}_{3}$

$85 \mathrm{R}^{1}=i \mathrm{Pr}$

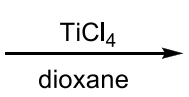

$87 \mathrm{Pr}^{i} \mathrm{H}$

$88 \quad \mathrm{CH}_{3} \quad \mathrm{CH}_{2} \mathrm{COOEt}$

$89 \mathrm{Pr}^{i} \quad \mathrm{CH}_{2}$ COOEt

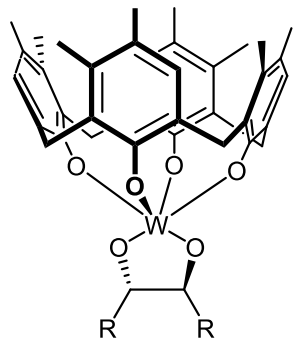

$R=P h$ or $R=-\left(\mathrm{CH}_{2}\right)_{4}^{-}$

90

Scheme 15. Condensation of benzylic alcohols 84 and 85 and their further functionalisation as esters ( $\mathbf{8 8}$ and $\mathbf{8 9}$ ) and diastereomeric derivatives $\mathbf{9 0 .}$

The more synthetically useful $\mathrm{X}_{4}$-methylcalix[4]arene $\mathbf{9 3} 49$ was prepared by condensation of 4-bromo-2-(hydroxymethyl)-5-methylphenol (91) and subsequent debromination of the calixarene 92 with $\mathrm{Pd} / \mathrm{C} /$ formic acid. The authors demonstrated the potential application of this calixarene by conversion to the azo ( $45 \%$ yield), tetrachloromethyl (88\%) and tetra(dimethylamino)methyl (75\%) derivatives, which have all been applied to the standard calixarenes previously.50 Addition of an adamantyl group to the upper rim failed and was attributed to the greater steric hinderance of the $\mathrm{X}_{4}$-methyl unlike the adamantyl derivative of the parent calixarene prepared by Khomich et al..51<smiles>Cc1cc(O)c(CO)cc1Br</smiles>

91<smiles>Cc1cc(Br)c(C)c(CC(C)(C)CC(=O)O)c1O</smiles>

92<smiles>Cc1ccc(C(C)(C)C)c(O)c1CC(C)(C)C</smiles>

93

Scheme 16. Condensation of benzylic alcohol 91 followed by de-bromination affords chiral calixarene $\mathbf{9 3}$ with a free reactive position for further functionalisation.

A very important member of the $C_{4}$ dissymmetric calixarenes is the calix[4]naphthalenes. The calix[4]naphthalene with 'endo' OH groups (see 94, Figure 22) was first prepared by Böhmer in $5 \%$ yield by the condensation of 1hydroxymethyl-2-naphthol with titanium tetrachloride in dioxane.40 The synthesis of the calix[4]naphthalene with 'exo' $\mathrm{OH}$ groups (95) was first reported by 
Georghiou.52 Condensation of 1-naphthol with formaldehyde in the presence of potassium carbonate as a catalyst yields the calix[4]naphthalene in $9.6 \%$ yield along with the corresponding isomers 96 and 97 in 16 and 5\% respectively. The most notable application of the calix[4]naphthalenes has been the complexation of [60]fullerene.53 Georghiou and coworkers have investigated these complexes in some detail.23,54

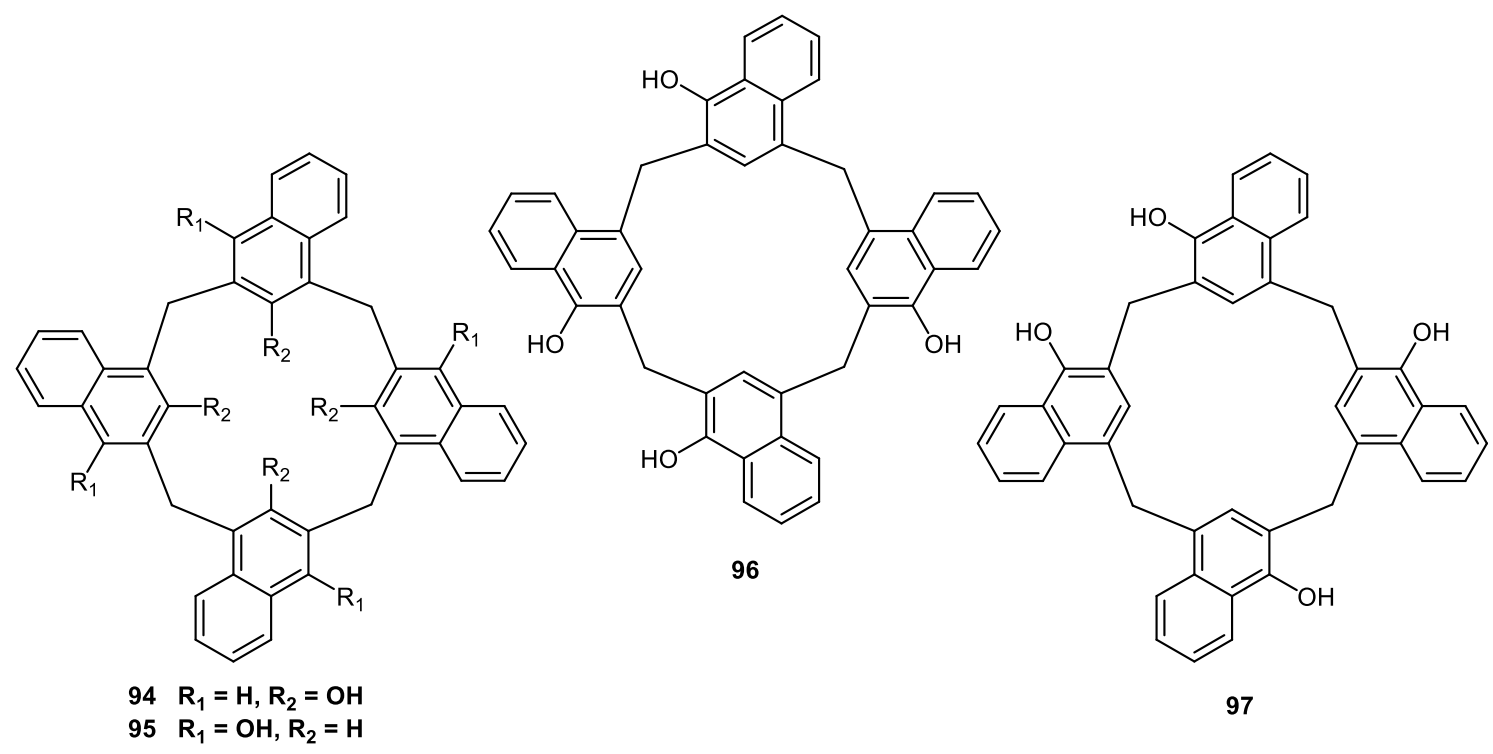

Figure 22. Calix[4]naphthalene regioisomers

\section{Resorcinarene based}

Of all of the helically chiral calixarene and resorcinarene compounds it is the $C_{4}$ dissymmetric resorcinarenes that are the most well studied of the series. The preparation of these can be divided into two categories, (i) the generation of chirality by the regioselective placement of groups about the resorcinarene upper rim, (ii) by the regioselective formation of the resorcinarene skeleton using resorcinol monoethers.

The tetra-benzoxazine derivatives make up a substantial portion of the first category (Figure 23). The first examples of this $(\mathbf{9 8}-\mathbf{1 0 0})$ were prepared by Matsushita and Matsui..55 Using achiral amines and aqueous formaldehyde solution they produced the corresponding racemic mixtures of benzoxazine functionalised resorcinarenes. 
However, no analytical details were reported and no mention of the chirality was made.

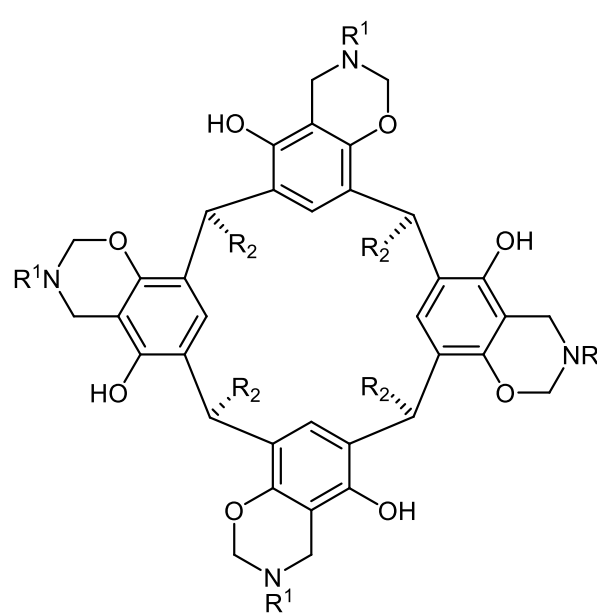

\begin{tabular}{|c|c|}
\hline & $\mathbf{R}_{\mathbf{1}}$ \\
\hline 98 & cyclohexyl \\
\hline 99 & $\mathrm{CH}_{2} \mathrm{COOEt}$ \\
\hline 100 & $\mathrm{Ph}$ \\
\hline 101 & $\mathrm{CH}_{2} \mathrm{Ph}$ \\
\hline 102 & mesityl \\
\hline 103 & $\mathrm{CH}_{3}$ \\
\hline 104 & $\mathrm{C}_{2} \mathrm{H}_{5}$ \\
\hline 105 & $\mathrm{C}_{3} \mathrm{H}_{7}$ \\
\hline 106 & $i \mathrm{Pr}$ \\
\hline 107 & $n \mathrm{Bu}$ \\
\hline 108 & $t \mathrm{Bu}$ \\
\hline 109 & $\mathrm{C}_{10} \mathrm{H}_{21}$ \\
\hline 110 & allyl \\
\hline 111 & $\mathrm{CH}_{2} \mathrm{Ph}$ \\
\hline $\begin{array}{l}112 \\
113\end{array}$ & $\begin{array}{l}\text { 1-adamantyl } \\
p-\mathrm{C}_{6} \mathrm{H}_{4} \mathrm{NO}_{2}\end{array}$ \\
\hline
\end{tabular}

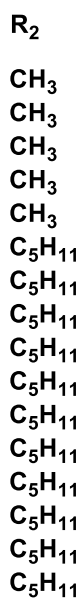

$\mathbf{R}_{1}$

$114 p-\mathrm{C}_{6} \mathrm{H}_{4} \mathrm{OC}_{6} \mathrm{H}_{5}$ $1153,4-\mathrm{C}_{6} \mathrm{H}_{3}(\mathrm{OMe})_{2}$ 116 2-(N-morpholino)ethyl $\mathrm{C}_{5} \mathrm{H}_{11}$ 117 2-methoxyethyl $\quad \mathrm{C}_{5} \mathrm{H}_{11}$ $118 \mathrm{CH}_{2} \mathrm{CH}_{2} \mathrm{Ph} \quad \mathrm{C}_{5} \mathrm{H}_{11}$ 119 mesityl $\quad \mathrm{C}_{5} \mathrm{H}_{11}$ $120 \mathrm{nBu} \quad \mathrm{C}_{11} \mathrm{H}_{23}$ $121 \mathrm{CH}_{2} \mathrm{Ph}$ 122 1-adamantyl $123 p-\mathrm{C}_{6} \mathrm{H}_{4} \mathrm{NO}_{2}$ $124 p-\mathrm{C}_{6} \mathrm{H}_{4} \mathrm{OC}_{6} \mathrm{H}_{5}$ $125\left(\mathrm{CH}_{2}\right)_{2} \mathrm{OH}$ $126\left(\mathrm{CH}_{2}\right)_{2} \mathrm{OH}$ $127\left(\mathrm{CH}_{2}\right)_{2} \mathrm{OH}$ $128\left(\mathrm{CH}_{2}\right)_{4} \mathrm{OH}$ $129\left(\mathrm{CH}_{2}\right)_{6} \mathrm{OH}$
$\mathbf{R}_{\mathbf{2}}$

$\mathrm{C}_{5} \mathrm{H}_{11}$ $\mathrm{C}_{5} \mathrm{H}_{11}$ $\mathrm{C}_{5} \mathrm{H}_{11}$ $\mathrm{C}_{11} \mathrm{H}_{23}$ $\mathrm{C}_{11} \mathrm{H}_{23}$ $\mathrm{C}_{11} \mathrm{H}_{23}$ $\mathrm{C}_{11} \mathrm{H}_{23}$ $\mathrm{C}_{2} \mathrm{H}_{5}$ $\mathrm{C}_{5} \mathrm{H}_{11}$ $\mathrm{C}_{11} \mathrm{H}_{23}$ $\mathrm{C}_{5} \mathrm{H}_{11}$ $\mathrm{C}_{11} \mathrm{H}_{23}$

Figure 23 - The formation of tetrabenzoxazine derivatives from achiral amines.

Further work by Böhmer56 with achiral amines (compounds 107, 111-114, 120 124) made full recognition of the chirality of the $C_{4}$ dissymmetric species and the potential for regioisomers. However 1 H n.m.r. spectroscopy with Pirkle's reagent produced no doubling of peaks and attempts at resolution failed until some years later.57 This was ascribed to the epimerisation of the benzoxazine ring via the iminium species (Figure 24). Resolution of several benzoxazine derivatives was achieved by HPLC57 on a Whelk O-1 chiral stationary phase, however, baseline separation was only achieved for $\mathbf{1 0 1}$ at low temperatures $\left(\mathrm{T}=-3{ }^{\circ} \mathrm{C}\right)$ to minimise the epimerisation (Daicel ChiralPak AD). The compounds produced by Bohmer and collaborators $(\mathbf{1 0 1}-\mathbf{1 2 4})$ and their syntheses have been reviewed.58 The energy barrier for interconversion of the enantiomers was calculated later by Trapp et al. to be $\Delta G \#(298 \mathrm{~K})=92 \pm 2 \mathrm{~kJ}$ mol-1.59

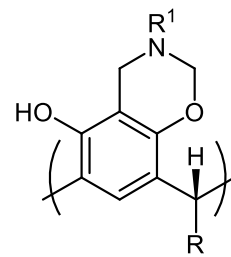<smiles>C=C</smiles>

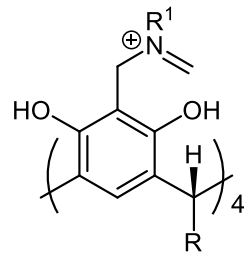<smiles>C=C</smiles>

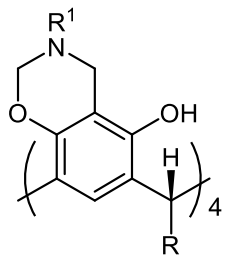

Figure 24. Proposed mechanism for the epimerisation of the benzoxazine rings. 
Similar preparations of benzoxazine derivatives using aminoalcohols (Figure 23, 125 - 129) provide an extended cavity with potentially useful functionality about the upper rim.60

The preparation of the benzoxazine derivatives using a variety of chiral amines was reported independently by Böhmer et al.56 $(\mathbf{1 3 0}-\mathbf{1 3 2}, \mathbf{1 3 6})$, Heaney et al.61 $(\mathbf{1 3 0}, 133$ - 139) and Iwanek et al.62 (134 and 138). When chiral amines were employed they acted as a chiral auxiliary and directed the ring closure reaction thereby enhancing the production of one diastereomer relative to the other. Thus, application of the appropriate $(R)$ - amines afforded the dextro-rotary tetrakisbenzoxazines $\mathbf{1 3 0}$ - 135 while the $(S)$ - amines afford the laevo-rotary enantiomers $(\mathbf{1 3 6}-\mathbf{1 3 9})$. This method of chiral resorcinarene preparation is particularly efficient as it requires no enantioselective separations. However, as the benzoxazine ring is labile, epimerisation still occurs. The three groups investigated the diastereomeric mixtures produced by acid catalysed epimerisation of the benzoxazine ring by n.m.r. spectroscopy. They also all produced compounds of good diastereomeric purity using $R$-(+)- and $S$-(-)- $\alpha$-methylbenzylamine using differing resorcinarene substrates.

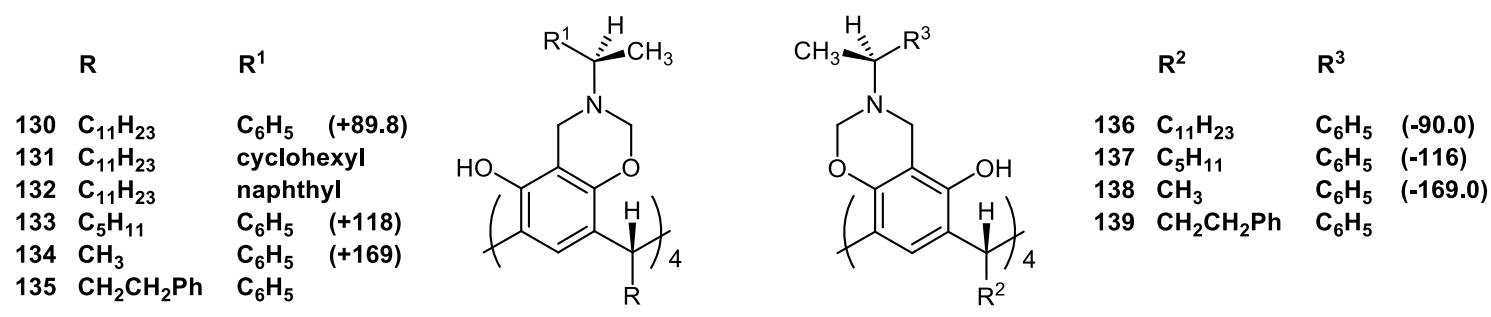

Figure 25. Resorcinarene tetrabenzoxazine derivatives produced using chiral amines.

The absolute stereochemistry of compounds 133 and 134 were determined using Xray methods by Heaney et al. and Iwanek et al. respectively.

Compound 138 was applied by Smith et al.63 as a chiral n.m.r. spectroscopy additive however, no significant interaction with a variety of guest molecules, was observed. Preparation of the first stable, enantiomerically pure helically chiral resorcinarene was achieved by Heaney et al.64,65 By methylation of the diastereomerically pure benzoxazine derivatives (130, 133 and 135 and respective enantiomers) using 
butyllithium and dimethylsulfate (or methyl triflate), racemisation can no longer occur $(140-142$, only (+) enantiomers shown). The chiral auxiliary may then be removed and useful transformations realised (scheme 17).

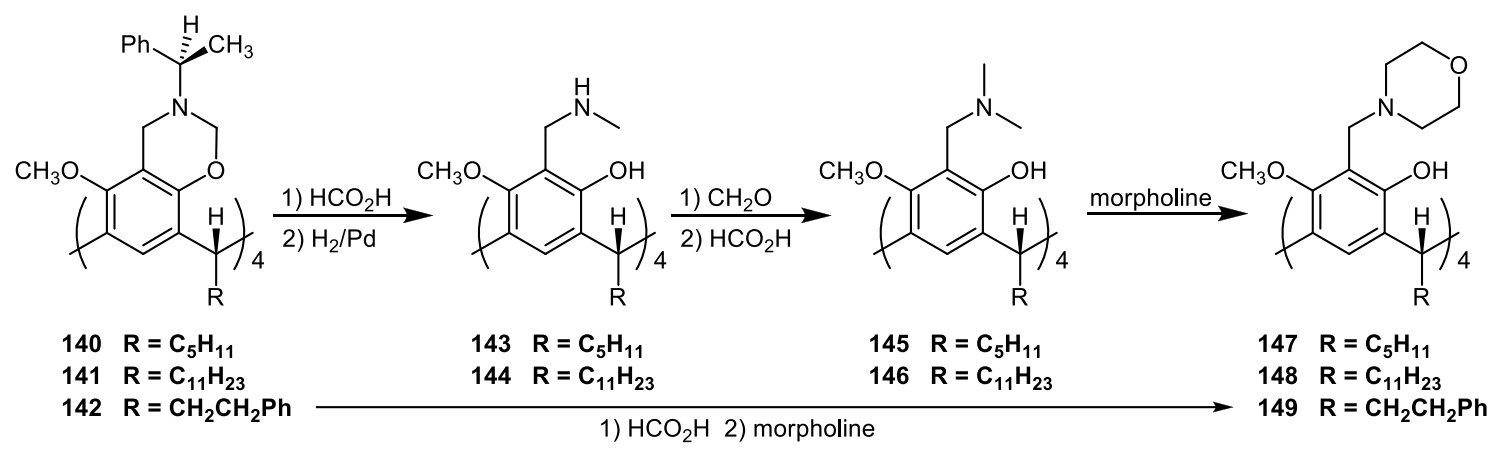

Scheme 17. Further transformations applied to single enantiomers of the resorcinarene tetrabenzoxazine derivatives.

The Mannich base theme was continued in 2002 by Iwanek66 who combined the Lprolinol derived $\mathbf{1 5 0}$ and phenylboronic acid to obtain a mixture of the boronheterocycle diastereomers 151 and 152.<smiles>[Z4]C(C)CC(C)Cc1cc(CCC)c(O)c(CN2CCC[C@H]2CO)c1O</smiles>

150

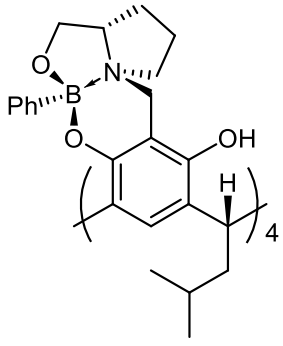

151<smiles>CC(C)CC(C)(C)c1cc(C(C)C)c(O)c2c1O[B-]1(c3ccccc3)OCC3CCC(C2)N31</smiles>

152

Scheme 18 - Preparation of chiral derivatives 151 and 152 from the L-prolinol derived mannich base $\mathbf{1 5 0}$.

These structurally rigid derivatives were formed as a mixture $(80: 20, \mathbf{1 5 1} / \mathbf{1 5 2})$ which could be separated by multiple fractional crystallisations. Both diastereomers were characterised by x-ray crystallography. 
The methodology was expanded later67 to the L-proline derivatives described in figure 26. All of the compounds were prepared in good yield and $>98 \%$ de.

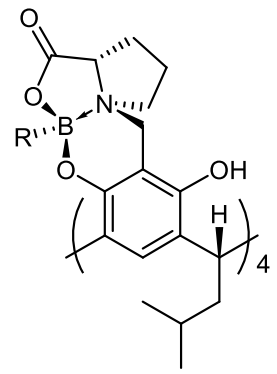

$153 \mathrm{R}=\mathrm{Ph}$

$154 \mathrm{R}=\mathrm{Et}$

$155 \mathrm{R}=\mathrm{OCH}_{3}$

$156 \mathrm{R}=\mathrm{OH}$

Figure 26. Chiral derivatives 153 - 156 prepared from the corresponding L-proline derived mannich base.

Somewhat related to the resorcinarene benzoxazines is the phenolic lactone acetal 158 produced by Cram.68 Compound 158 and its enantiomer were prepared in $18 \%$ yield by the acid catalysed rearrangement of the cavitand 157 .

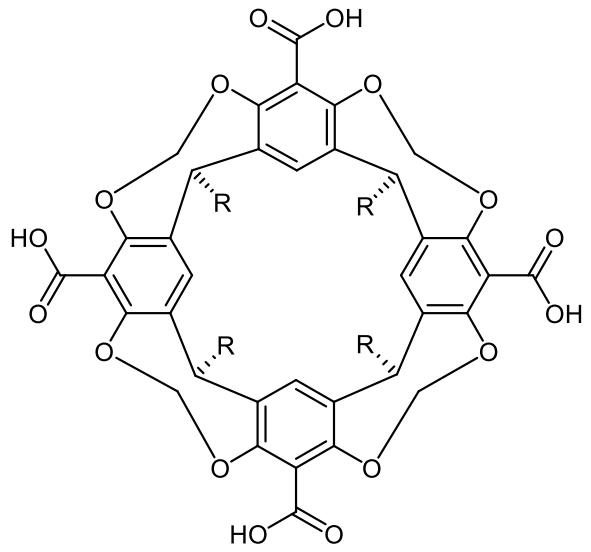

157

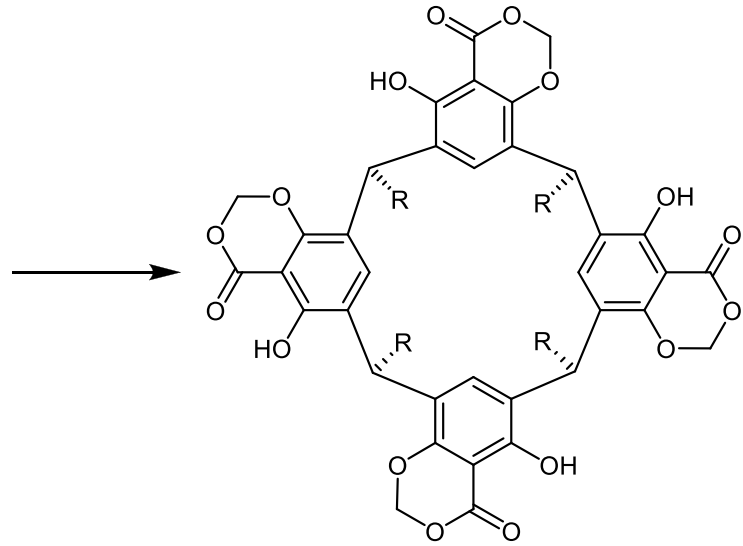

158

Scheme 19. Acid catalysed rearrangement of cavitand 157 to afford the $C_{4}$ dissymmetric derivative $\mathbf{1 5 8 .}$

A very interesting example of regioselective placement of groups about the resorcinarene cavity to generate $C_{4}$ dissymmetry is that given in scheme 20 . Terent'eva and coworkers69 produced the $C_{4}$ isomer 161 by reaction of 2-ethyl-1,3,2benzoxaphospholine 159 with the tetra(dimethylaminomethyl)calix[4]-resorcinarene 160. This reaction is especially curious since alkylation with the majority of 
alkylating agents produce a complex mixture of regioisomers or in the case of reagents such as toluenesulfonyl chloride, produces the distal tetratosylate. 70 No ${ }_{13} \mathrm{C}$ n.m.r data was provided for $\mathbf{1 6 1}$ and the $1 \mathrm{H}$ n.m.r. spectroscopic data provided is insufficient for a conclusive assignment.

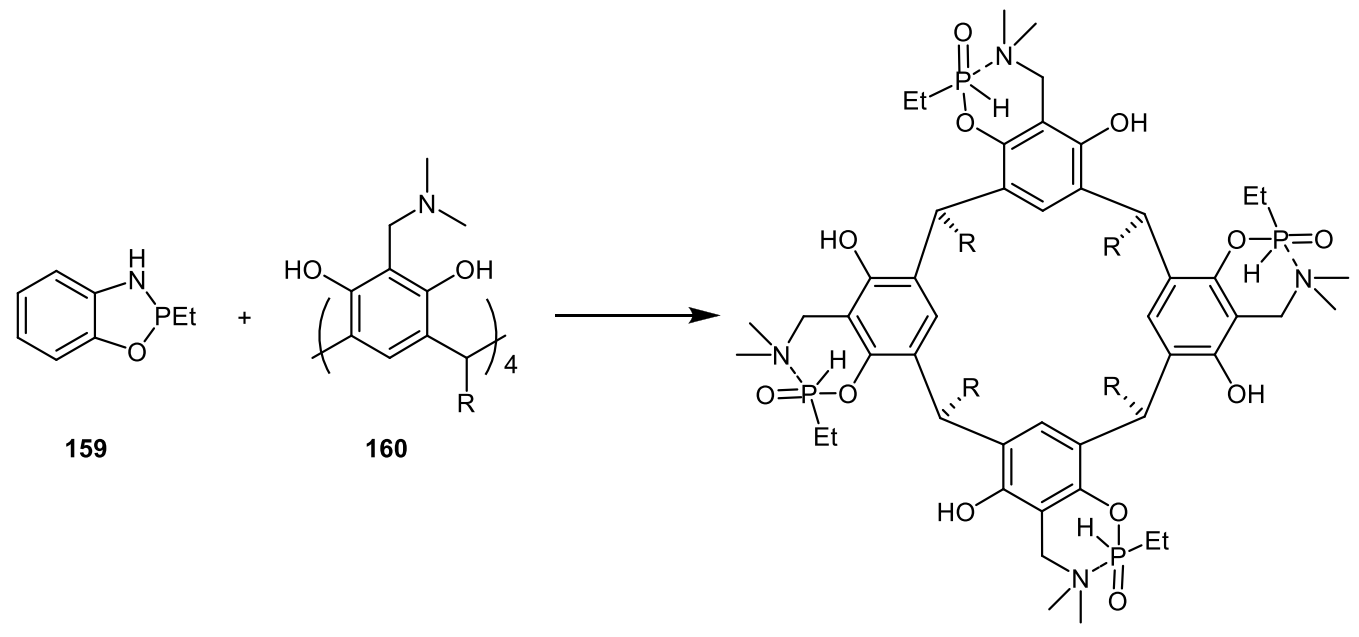

161

Scheme 20. Synthesis and proposed structure of the phosphorus based resorcinarene derivative 161.

We reported the first example of a regioselective formation of the resorcinarene skeleton from resorcinol mono-ethers.71 The condensation of the mono-ethers $\mathbf{1 6 2}$ and 164 with alkyl aldehydes in the presence of boron trifluoride etherate gave the respective $C_{4}$ dissymmetric resorcinarenes $164-166$ as their racemates in high yields (scheme 21). This synthesis is rather remarkable as it involves the condensation of eight individual molecules that combine in a completely regioselective manner despite the possibilities. The chirality was demonstrated by $1 \mathrm{H}$ n.m.r spectroscopy with the chiral shift reagent tris[3-(heptafluoropropylhydroxymethylene)-(+)-camphorate] which caused doubling of the aromatic, phenolic and methine signals. The partial separation of resorcinarene 164 was also achieved by enantioselective HPLC (Daicel Chiralpak AD hexane/2-propanol 98:2). An x-ray crystal structure for $\mathbf{1 6 4}$ was obtained which confirmed the dissymmetric arrangement of the resorcinarene upper rim. 

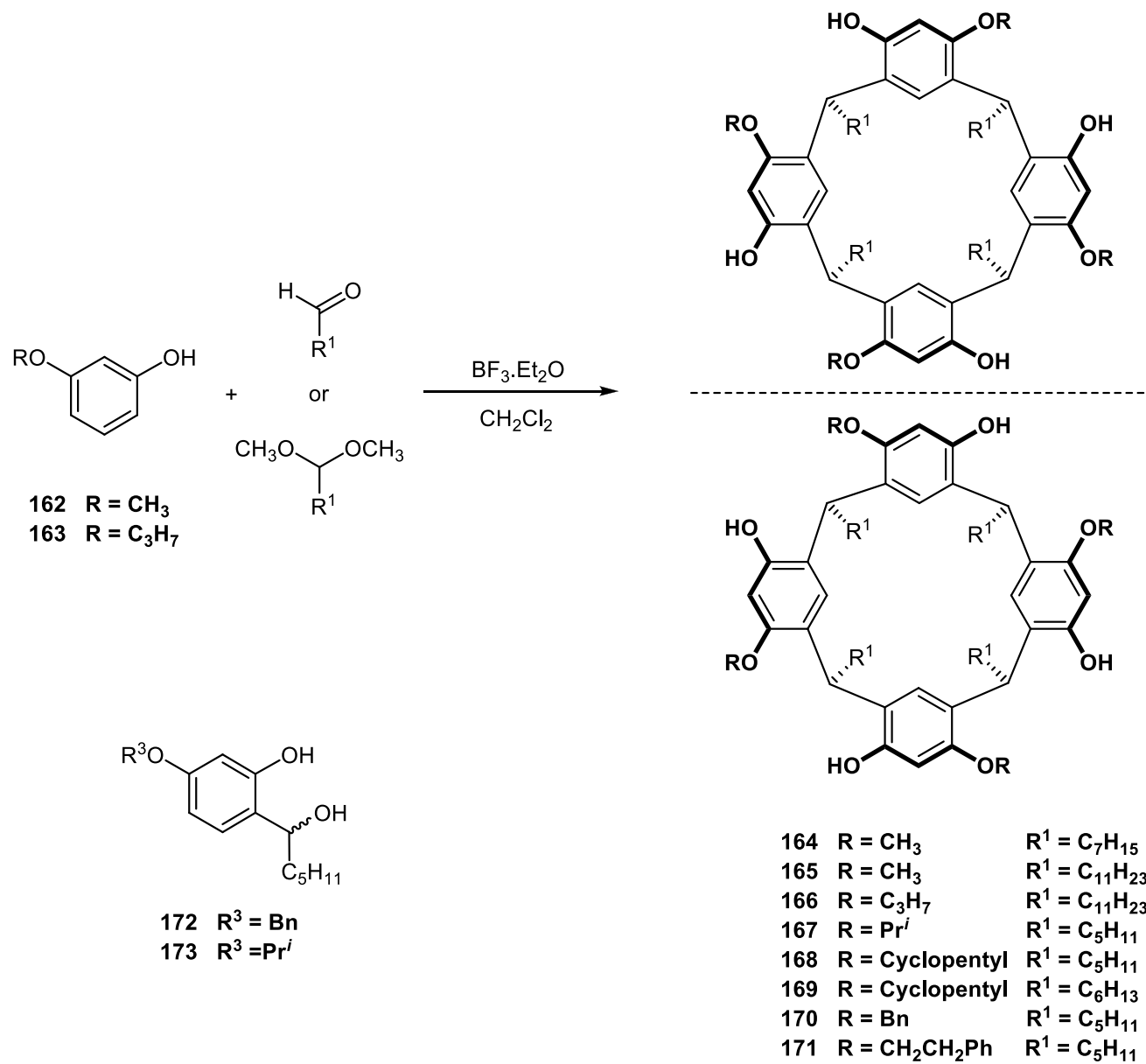

Scheme 21. The synthesis of $C_{4}$ dissymmetric resorcinarenes by condensation of resorcinol mono-ethers and alkyl aldehydes.

Heaney et al. further expanded the series with the preparation of 167 - 171.65 They found that the tetracyclopentyloxy and tetrabenzyloxy resorcinarenes $\mathbf{1 6 8}-\mathbf{1 7 0}$ could not be prepared by the standard boron trifluoride / aldehyde protocol presumably due to instability of the reaction intermediate. By application of the aldehyde dimethyl acetals rather than the aldehydes the resorcinarenes were afforded in moderate to excellent yields. In the same publication the presumed reaction intermediates (172 and 173) were prepared and studied as useful precursors to the resorcinarenes. In particular the proposed intermediate 173 in the presence of boron trifluoride etherate did indeed give the desired resorcinarene (167) in $82 \%$ yield. The facile preparation of the $C_{4}$ dissymmetric resorcinarenes was made all the more potent by Heaney with the publication of a simple synthesis for resorcinol monoethers. 65 
The first resolution of a $C_{4}$ dissymmetric resorcinarene prepared by this method was achieved by Mattay and coworkers.72 By monofunctionalisation of the resorcinarene 174 with a camphorsulfonyl moiety, the resulting diastereomers could be resolved by HPLC. Hydrolysis of the sulfonate esters $\mathbf{1 7 5 a}$ and $\mathbf{1 7 5 b}$ yielded the pure resorcinarene enantiomers. Unfortunately the absolute stereochemistry of the resorcinarenes was not determined.

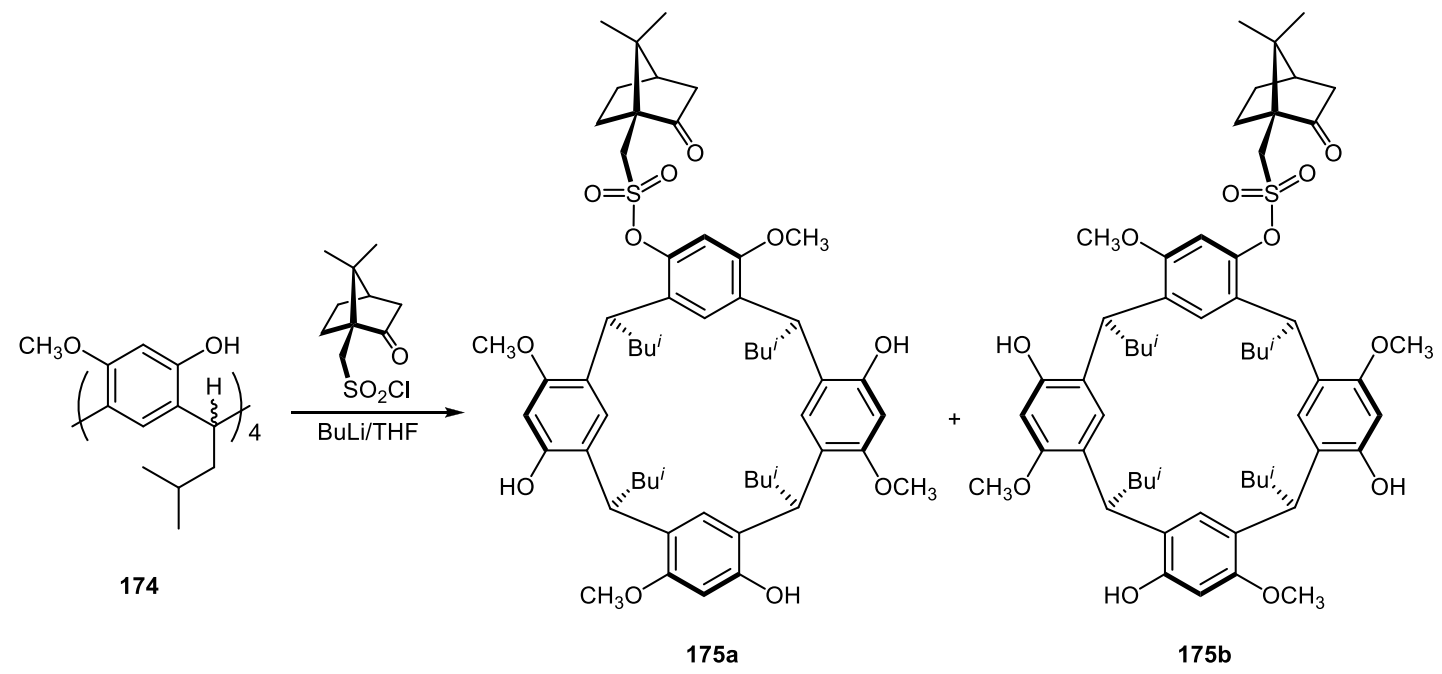

Scheme 22. Resolution of chiral tetramethoxyresorcinarene by formation of the diastereomeric mono-camphorsulfonate esters.

They also applied the boron trifluoride protocol (in the same publication) to the chiral resorcinol mono-ether $\mathbf{1 7 6}$ so as to recover the resorcinarenes as diastereomeric mixtures (see Scheme 23) which could be separated by HPLC. Resorcinarene formation with $\mathbf{1 7 6}$ and 3-methyl butanal appeared to favour the formation of the laevorotary diastereomer while dodecanal produced a 1:1 mixture of diastereomers (178a and 178b). Again, no X-ray data was provided for any of the diastereomers and the absolute stereochemistry was not elucidated at the time.<smiles>CC[C@H](C)COc1cccc(O)c1</smiles>
176<smiles>[R]C=O</smiles>

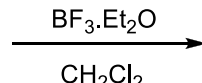
$\mathrm{CH}_{2} \mathrm{Cl}_{2}$

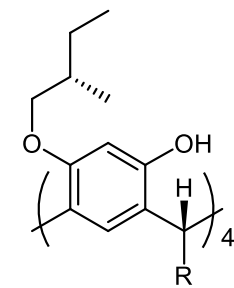

177a $\mathrm{R}=\mathrm{Bu}^{i}$ 178a $R=C_{11} H_{23}$<smiles>[R]C(C)(C)c1cc(C(C)C)c(O)cc1OC[C@@H](C)CC</smiles>

177b $\mathrm{R}=\mathrm{Bu}^{i}$ 178b $\mathrm{R}=\mathrm{C}_{11} \mathrm{H}_{23}$ 
Scheme 23. Preparation of diastereomeric resorcinarenes by condensation of a chiral resorcinol mono-ether (176) with alkyl aldehydes.

The issue of the absolute stereochemistry was addressed by Mattay et al. in 2005.73 They converted the resolved enantiomers $\mathbf{1 7 4 a}$ and $\mathbf{1 7 4 b}$ in to the $(S)-(-)-(\alpha)$ methylbenzylamine derived diastereomeric amide derivatives 180a and 180b. A single crystal X-ray structure of $\mathbf{1 8 0 a}$ was obtained and the absolute stereochemistry of the laevorotary enantiomer of $\mathbf{1 7 4}$ assigned the all $(S)$ configuration by relation to $180 a$.

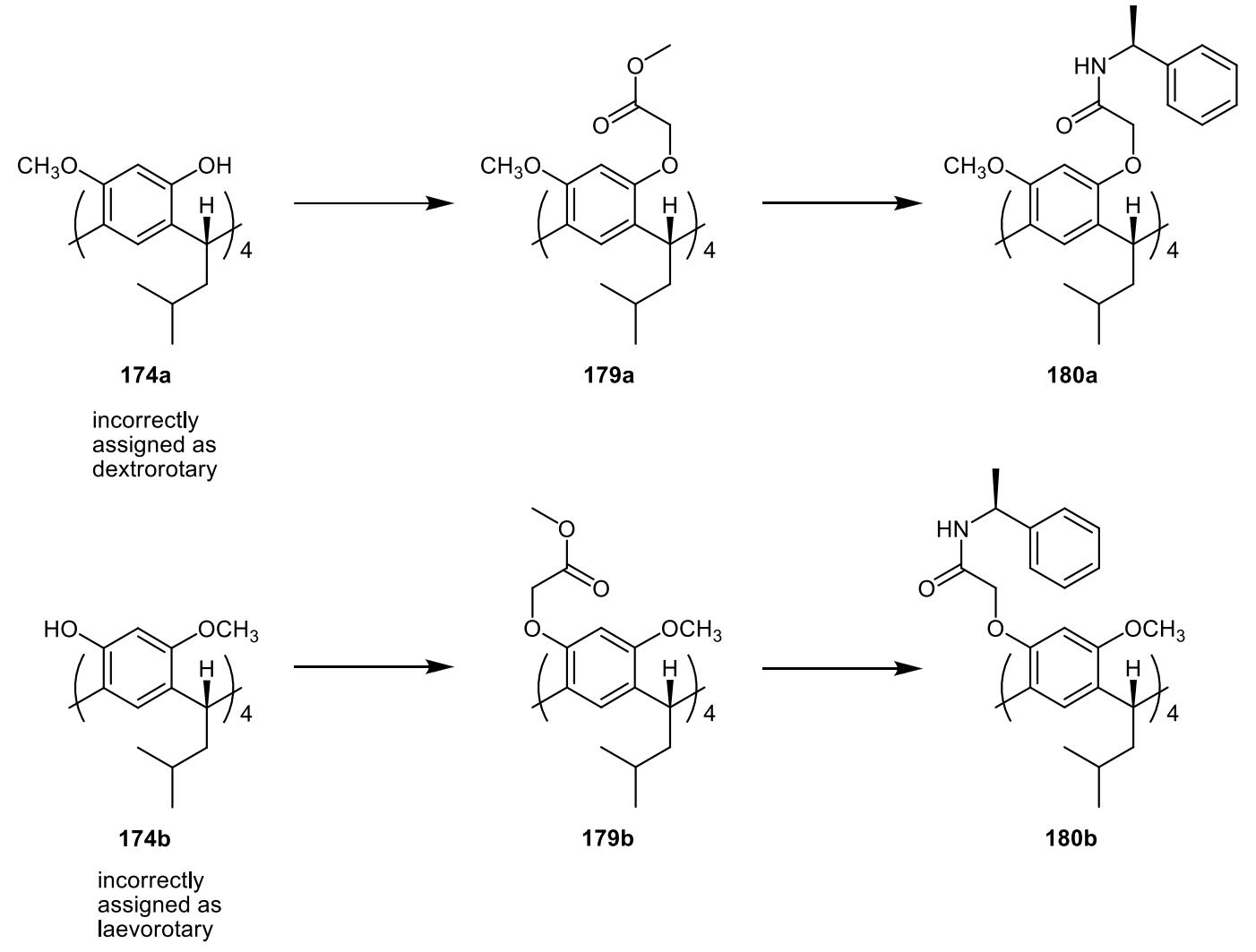

Scheme 24. Synthesis of the diastereomeric amide derivatives $\mathbf{1 8 0}$ by amidation of the tetraesters 179 with $(S)-(-)-(\alpha)$-methylbenzylamine.

We reported 21 gram scale syntheses of the amides $\mathbf{1 8 2}-\mathbf{1 8 4}$ by an alternate route using the enantio-pure alkylating agent derived from $(R)-(+)-(\alpha)$-methylbenzylamine. These amide functionalised resorcinarenes were readily resolved to their respective diastereomers in good yields demonstrating the generality of the resolution process. A single crystal x-ray structure of $\mathbf{1 8 2} \mathbf{a}$ was obtained and the absolute 
stereochemistry of the first eluting diastereomer was found to be the all $(R)$ configuration at the resorcinarene stereocentres, in good accord with Mattay's findings.
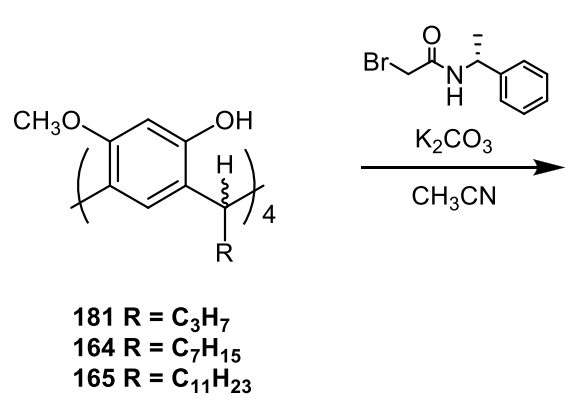<smiles>CCCCC(C)(C)c1cc(CC)c(OCC(=O)NC(C)c2ccccc2)cc1OC</smiles>

182a $\mathrm{R}=\mathrm{C}_{3} \mathrm{H}_{7}$

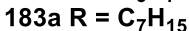

184a $R=C_{11} H_{23}$<smiles>CCCCC(C)(C)c1cc(C(C)C)c(OC)cc1OCC(=O)N[C@H](C)c1ccccc1</smiles>

$182 \mathrm{~b} \mathrm{R}=\mathrm{C}_{3} \mathrm{H}_{7}$ 183b R $=\mathrm{C}_{7} \mathrm{H}_{15}$ $184 b \mathrm{R}=\mathrm{C}_{11} \mathrm{H}_{23}$

Scheme 25. Synthesis of diastereomeric amide derivatives by attachment of a chiral alkylating agent.

The absolute stereochemistry for several resorcinarene enantiomers, including 174, was elucidated by X-ray crystallography in 200674 by the synthesis and resolution of the tetracamphorsulfonates (Scheme 26).<smiles>[R]C(C)(C)c1cc(C(C)(C)C)c(O)cc1OC</smiles>

$185 \mathrm{R}=\mathrm{C}_{5} \mathrm{H}_{11}$ $174 \mathrm{R}=\mathrm{Bu}^{i}$

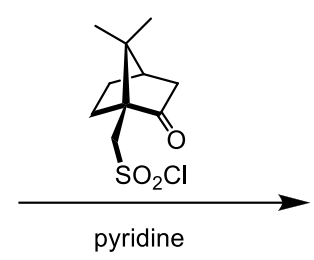

pyridine

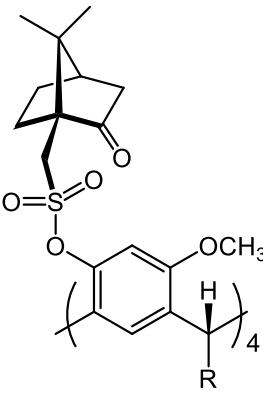

186a $\mathrm{R}=\mathrm{C}_{5} \mathrm{H}_{11}$ $187 \mathrm{a} R=\mathrm{Bu}^{i}$<smiles>[R]C(C)(C)c1cc(C(C)C)c(OC)cc1OS(=O)(=O)CC12CCC(CC1=O)C2(C)C</smiles>

$186 \mathrm{~b} \mathrm{R}=\mathrm{C}_{5} \mathrm{H}_{11}$ $187 \mathrm{~b} \mathrm{R}=\mathrm{Bu}^{i}$

Scheme 26. Synthesis of the resorcinarene tetracamphorsulfonate esters with the absolute stereochemistry for the first eluting (series a) and second eluting (series b) diastereomers.

Having determined the absolute stereochemistry of the tetracamphorsulfonate esters the ester functionality was removed by alkaline hydrolysis to obtain the pure enantiomers for which the absolute stereochemistry of the dextrorotary 
resorcinarenes 185a and 174a were of the all $(S)$ - configuration as shown in scheme 27. This result contradicted Mattay's previous assignment, however, the evidence is strongly in favour of our conclusion and has been corroborated by Mattay74.

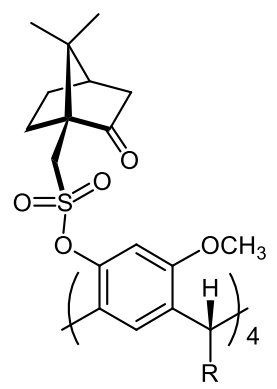

$186 \mathrm{a} R=\mathrm{C}_{5} \mathrm{H}_{11}$ $187 \mathrm{a} \mathbf{R}=\mathrm{Bu}^{i}$

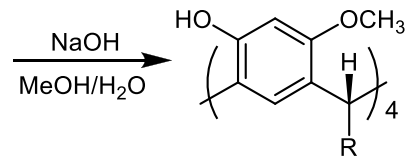

$(+)-185 \mathrm{a} R=\mathrm{C}_{5} \mathrm{H}_{11}$ $(+)-174 \mathrm{a} \mathrm{R}=\mathrm{Bu}^{i}$

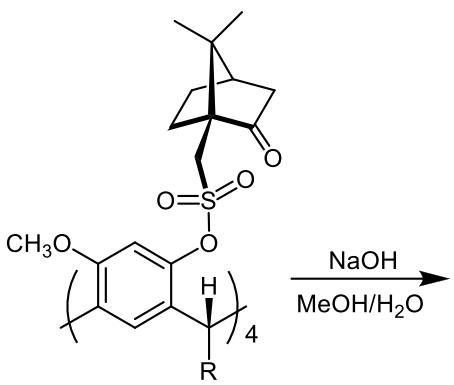

186b R $=\mathrm{C}_{5} \mathrm{H}_{11}$ $187 \mathrm{~b} \mathrm{R}=\mathrm{Bu}^{i}$<smiles>[R]C(C)(C)c1cc(C(C)(C)C)c(OC)cc1O</smiles>

$(-)-185 b \mathrm{~B}=\mathrm{C}_{5} \mathrm{H}_{11}$ $(-)-174 \mathrm{~b} R=\mathrm{Bu}^{i}$

Scheme 27. Hydrolysis of the camphorsulfonate esters to afford enantio-pure chiral resorcinarenes.

During the course of the camphorsulfonylation studies a number of di- and tricamphorsulfonates were also observed. In particular when resorcinarenes with larger ether groups at the upper rim such as $\mathbf{1 6 7}$ and $\mathbf{1 6 8}$ were combined with camphorsulfonyl chloride, the mono- or di-esters could be obtained depending on the base and conditions applied.

The absolute stereochemistry of the resorcinarenes was corroborated 21 by conversion into their tetrabenzoxazine derivatives (such as $\mathbf{1 8 8}$, Scheme $\mathbf{2 8}$ ).<smiles>CCc1cc(C(C)(C)CC(C)C)c(OC)cc1O</smiles>

$174 a$

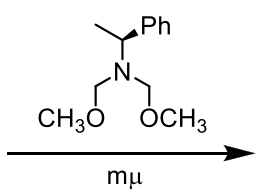

$\mathrm{m}$<smiles>COc1c(C(C)C)cc(C(C)C)c2c1CN(C(C)c1ccccc1)CO2</smiles>

188

Scheme 28. Synthesis of the diastereomerically pure tetrabenzoxazine derivative 188. 
The absolute stereochemistry of $\mathbf{1 8 8}$ had been determined by x-ray crystallography in a previous study regarding the functionalisation and resolution of the resorcinarenes described in scheme 29 as their tetrabenzoxazine derivatives 140, $191-198.21$
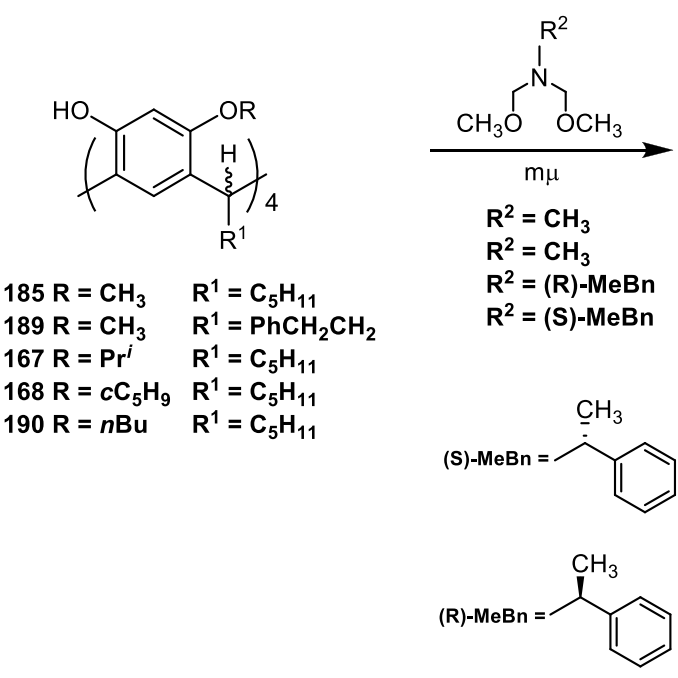<smiles>[R20]c1c(C([R])(C)C)cc(C([R])(C)C)c2c1CN([R])CO2</smiles>

$+$

$\begin{array}{lll}191 \mathrm{R}=\mathrm{CH}_{3} & \mathrm{R}^{1}=\mathrm{C}_{5} \mathrm{H}_{11} & \mathrm{R}^{2}=\mathrm{Bn} \\ 192 \mathrm{R}=\mathrm{CH}_{3} & \mathrm{R}^{1}=\mathrm{C}_{5} \mathrm{H}_{11} & \mathrm{R}^{2}=\mathrm{CH}_{3} \\ 193 \mathrm{R}=\mathrm{CH}_{3} & \mathrm{R}^{1}=\mathrm{PhCH}_{2} \mathrm{CH}_{2} \mathrm{R}^{2}=\mathrm{CH}_{3} \\ 140 \mathrm{R}=\mathrm{CH}_{3} & \mathrm{R}^{1}=\mathrm{C}_{5} \mathrm{H}_{11} & \mathrm{R}^{2}=(\mathrm{R})-\mathrm{MeBn} \\ 194 \mathrm{R}=\mathrm{Pr}^{i} & \mathrm{R}^{1}=\mathrm{C}_{5} \mathrm{H}_{11} & \mathrm{R}^{2}=(\mathrm{R})-\mathrm{MeBn} \\ 195 \mathrm{R}=\mathrm{cC}_{5} \mathrm{H}_{9} & \mathrm{R}^{1}=\mathrm{C}_{5} \mathrm{H}_{11} & \mathrm{R}^{2}=(\mathrm{R})-\mathrm{MeBn} \\ 196 \mathrm{R}=\mathrm{CH}_{3} & \mathrm{R}^{1}=\mathrm{C}_{5} \mathrm{H}_{11} & \mathrm{R}^{2}=(\mathrm{S})-\mathrm{MeBn} \\ 197 \mathrm{R}=\mathrm{Pr}^{i} & \mathrm{R}^{1}=\mathrm{C}_{5} \mathrm{H}_{11} & \mathrm{R}^{2}=(\mathrm{S})-\mathrm{MeBn} \\ 198 \mathrm{R}=n \mathrm{nu} & \mathrm{R}^{1}=\mathrm{C}_{5} \mathrm{H}_{11} & \mathrm{R}^{2}=(\mathrm{S})-\mathrm{MeBn}\end{array}$

Scheme 29. Preparation of several tetrabenzoxazine derivatives from the chiral $C_{4}$ dissymmetric resorcinarenes (note: $c \mathrm{C}_{5} \mathrm{H}_{9}=$ cyclopentyl).

During the course of those studies21 it was determined that the $C_{4}$ dissymmetric resorcinarenes could not be functionalised as the tetrabenzoxazines in the usual manner (cat. $\mathrm{NaOH}$, amine and paraformaldehyde). The failure was ascribed to the relatively weakly acidic phenols of the $C_{4}$ resorcinarenes which were determined to be approximately $2 \mathrm{pKa}$ units less acidic than the achiral octahydroxyresorcinarenes. However, by application of more forcing conditions or microwave irradiation, the tetrabenzoxazine derivatives could be obtained in good yield.

The chiral tetrabenzoxazines were examined as ligands for the addition of diethylzinc to benzaldehyde. In general only moderate \% ee's were obtained, the best being a conversion of $95 \%$ with an ee of $83 \%$ with compound 194 (in favour of (S)-(-)-1phenylpropanol).

\section{Outlook}

This review provides a summary of current work regarding the syntheses and application of calixarenes and resorcinarenes having $C_{\mathrm{n}}$ dissymmetry. It is not 
intended to be comprehensive but merely a guide to some of the major themes.

However, there is a vast array of modifications and applications yet still available to the aspiring macrocycle chemist. Chiral ligands for catalysis, enzyme mimicry and chiral separation science have barely been explored and offer much promise.

(1) Shirakawa, S.; Moriyama, A.; Shimizu, S. Eur. J. Org. Chem. 2008, 5957; Homden, D. M.; Redshaw, C. Chem. Rev. (Washington, DC, U. S.) 2008, 108, 5086; Frediani, M.; Semeril, D.; Mariotti, A.; Rosi, L.; Frediani, P.; Rosi, L.; Matt, D.; Toupet, L. Macromol. Rapid Commun. 2008, 29, 1554.

(2) Kivlehan, F.; Mace, W. J.; Moynihan, H. A.; Arrigan, D. W. M. Anal. Chim. Acta 2007, 585, 154; Pomecko, R.; Asfari, Z.; Hubscher-Bruder, V.; Bochenska, M.; Arnaud-Neu, F. Supramol. Chem. 2007, 19, 459; Schazmann, B.; Diamond, D. New J. Chem. 2007, 31, 587.

(3) Meyer, R.; Jira, T. Curr. Anal. Chem. 2007, 3, 161; Sliwka-Kaszynska, M. Crit. Rev. Anal. Chem. 2007, 37, 211.

(4) Sansone, F.; Barboso, S.; Casnati, A.; Fabbi, M.; Pochini, A.; Ugozzoli, F.; Ungaro, R. Eur. J. Org. Chem. 1998, 897.

(5) Budka, J.; Tkadlecova, M.; Lhotak, P.; Stibor, I. Tetrahedron 2000, 56, 1883.

(6) Favre, H. A.; Hellwinkel, D.; Powell, W. H.; Smith, H. A.; Tsay, S. S. C.

Pure Appl. Chem. 2002, 74, 809.

(7) Cahn, R. S.; Ingold, C.; Prelog, V. Angew. Chem., Int. Ed. Engl. 1966, 5, 385.

(8) Dodziuk, H.; Mirowicz, M. Tetrahedron: Asymmetry 1990, 1, 171.

(9) Vysotsky, M.; Schmidt, C.; Bohmer, V. Advances in Supramolecular Chemistry, Vol 7 2000, 7, 139.

(10) Dalla Cort, A.; Mandolini, L.; Pasquini, C.; Schiaffino, L. New J. Chem. 2004, 28, 1198.

(11) Cahn, R. S.; Ingold, C. K.; Prelog, V. Experientia 1956, 12, 81.

(12) Boehmer, V.; Marschollek, F.; Zetta, L. J. Org. Chem. 1987, 52, 3200.

(13) Ferguson, G.; Gallagher, J. F.; Giunta, L.; Neri, P.; Pappalardo, S.; Parisi, M. J. Org. Chem. 1994, 59, 42.

(14) Yakovenko, A. V.; Boyko, V. I.; Danylyuk, O.; Suwinska, K.; Lipkowski, J.; Kalchenko, V. I. Org. Lett. 2007, 9, 1183; Narumi, F.; Hattori, T.; Yamabuki, W.; Kabuto, C.; Kameyama, H. Tetrahedron: Asymmetry 2005, 16, 793; Luo, J.; Zheng, Q.-Y.; Chen, C.-F.; Huang, Z.-T. Chem.--Eur. J. 2005, 11, 5917; Li, S. Y.; Zheng, Q. Y.; Chen, C. F.; Huang, Z. T. Tetrahedron: Asymmetry 2005, 16, 641.

(15) Xu, Z.-X.; Li, G.-K.; Chen, C.-F.; Huang, Z.-T. Tetrahedron 2008, 64, 8668. (16) Xu, Z.-X.; Zhang, C.; Yang, Y.; Chen, C.-F.; Huang, Z.-T. Org. Lett. 2008, $10,477$.

(17) Xu, Z.-X.; Zhang, C.; Zheng, Q.-Y.; Chen, C.-F.; Huang, Z.-T. Org. Lett. 2007, 9, 4447.

(18) Prelog, V.; Helmchen, G. Angew. Chem. Int. Ed. Engl. 1982, 21, 567;

Helmchen, G. In Methods in Organic Chemistry 1995; Vol. E21a, p 1.

(19) Schlogl, K. Top. Curr. Chem. 1984, 125, 29.

(20) Moss, G. P. Pure Appl. Chem. 1996, 68, 2193.

(21) Buckley, B. R.; Boxhall, J. Y.; Page, P. C. B.; Chan, Y.; Elsegood, M. R. J.; Heaney, H.; Holmes, K. E.; McIldowie, M. J.; McKee, V.; McGrath, M. J.; 
Mocerino, M.; Poulton, A. M.; Sampler, E. P.; Skelton, B. W.; White, A. H. Eur. J. Org. Chem. 2006, 5117.

(22) Wzorek, A.; Gawdzik, B.; Urbaniak, M.; Iwanek, W. Recent Res. Dev. Org. Chem. 2004, 8, 85; Bohmer, V.; Kraft, D.; Tabatabai, M. J. Inclusion Phenom. 1994, $19,17$.

(23) Mizyed, S.; Ashram, M.; Miller, D. O.; Georghiou, P. E. J. Chem. Soc., Perkin Trans. 2 2001, 1916.

(24) Nakazaki, M. Top. Stereochem. 1984, 15, 199.

(25) Reddy, P. A.; Gutsche, C. D. J. Org. Chem. 1993, 58, 3245.

(26) Taghvaei-Ganjali, S.; Shafai, M.; Khosravi, M. Acta Chim. Slov. 2002, 49,

903.

(27) Verboom, W.; Bodewes, P. J.; Vanessen, G.; Timmerman, P.; Vanhummel, G. J.; Harkema, S.; Reinhoudt, D. N. Tetrahedron 1995, 51, 499.

(28) Otsuka, H.; Shinkai, S. J. Am. Chem. Soc. 1996, 118, 4271.

(29) No, K. H.; Gutsche, C. D. J. Org. Chem. 1982, 47, 2713; Kaemmerer, H.; Happel, G. Makromol. Chem. 1980, 181, 2049.

(30) Asao, T.; Ito, S.; Morita, N. Tetrahedron Lett. 1988, 29, 2839.

(31) Casabianca, H.; Royer, J.; Satrallah, A.; Taty-C, A.; Vicens, J. Tetrahedron Lett. 1987, 28, 6595.

(32) Ueda, Y.; Fujiwara, T.; Tomita, K. I.; Asfari, Z.; Vicens, J. J. Inclusion Phenom. 1993, 15, 341.

(33) Tabatabai, M.; Vogt, W.; Boehmer, V. Tetrahedron Lett. 1990, 31, 3295.

(34) Shinkai, S.; Arimura, T.; Kawabata, H.; Murakami, H.; Iwamoto, K. J. Chem. Soc., Perkin Trans. 1 1991, 2429.

(35) Andreetti, G. D.; Bohmer, V.; Jordon, J. G.; Tabatabai, M.; Ugozzoli, F.; Vogt, W.; Wolff, A. J. Org. Chem. 1993, 58, 4023.

(36) Ikeda, A.; Yoshimura, M.; Lhotak, P.; Shinkai, S. J. Chem. Soc., Perkin Trans. 1 1996, 1945.

(37) Agbaria, K.; Wohnert, J.; Biali, S. E. J. Org. Chem. 2001, 66, 7059.

(38) Konishi, H.; Tamura, T.; Okhkubo, H.; Kobayashi, K.; Morikawa, O. Chem. Lett. 1996, 685.

(39) Agena, C.; Wolff, C.; Mattay, J. Eur. J. Org. Chem. 2001, 2977.

(40) Wolff, A.; Boehmer, V.; Vogt, W.; Ugozzoli, F.; Andreetti, G. D. J. Org. Chem. 1990, 55, 5665.

(41) Kusano, T.; Tabatabai, M.; Okamoto, Y.; Boehmer, V. J. Am. Chem. Soc. 1999, 121, 3789; Pickard, S. T.; Pirkle, W. H.; Tabatabai, M.; Vogt, W.; Boehmer, V. Chirality 1993, 5, 310.

(42) Gutsche, C. D. Calixarenes; The Royal Society of Chemistry, Cambride, 1989.

(43) Bitter, I.; Grun, A.; Toth, G.; Balazs, B.; Horvath, G.; Toke, L. Tetrahedron 1998, 54, 3857.

(44) He, Y. B.; Xiao, Y. J.; Meng, L. Z.; Zeng, Z. Y.; Wu, X. J.; Wu, C. T.

Tetrahedron Lett. 2002, 43, 6249.

(45) Ikeda, A.; Shinkai, S. J. Chem. Soc., Perkin Trans. 1 1993, 2671.

(46) Shivanyuk, A.; Schmidt, C.; Bohmer, V.; Paulus, E. F.; Lukin, O.; Vogt, W. J. Am. Chem. Soc. 1998, 120, 4319.

(47) Salorinne, K.; Nissinen, M. Org. Lett. 2006, 8, 5473.

(48) Wu, T. T.; Speas, J. R. J. Org. Chem. 1987, 52, 2330.

(49) Fu, D. K.; Xu, B.; Swager, T. M. J. Org. Chem. 1996, 61, 802. 
(50) Shinkai, S.; Araki, K.; Shibata, J.; Manabe, O. J. Chem. Soc., Perkin Trans. 1 1989, 195; Almi, M.; Arduini, A.; Casnati, A.; Pochini, A.; Ungaro, R. Tetrahedron 1989, 45, 2177; Gutsche, C. D.; Nam, K. C. J. Am. Chem. Soc. 1988, 110, 6153.

(51) Khomich, A. N.; Shokova, E. A.; Kovalev, V. V. Synlett 1994, 1027.

(52) Georghiou, P. E.; Li, Z. Tetrahedron Lett. 1993, 34, 2887; Georghiou, P. E.;

Li, Z. J. Inclusion Phenom. 1994, 19, 55.

(53) Georghiou, P. E.; Mizyed, S.; Chowdhury, S. Tetrahedron Lett. 1999, 40,

611.

(54) Mizyed, S.; Georghiou, P. E.; Ashram, M. J. Chem. Soc., Perkin Trans. 2 2000, 277.

(55) Matsushita, Y.; Matsui, T. Tetrahedron Lett. 1993, 34, 7433.

(56) Arnecke, R.; Boehmer, V.; Friebe, S.; Gebauer, S.; Krauss, G. J.; Thondorf, I.; Vogt, W. Tetrahedron Lett. 1995, 36, 6221.

(57) Bohmer, V.; Caccamese, S.; Principato, G.; Schmidt, C. Tetrahedron Lett. 1999, 40, 5927.

(58) Airola, K.; Bohmer, V.; Paulus, E. F.; Rissanen, K.; Schmidt, C.; Thondorf, I.; Vogt, W. Tetrahedron 1997, 53, 10709.

(59) Trapp, O.; Caccamese, S.; Schmidt, C.; Bohmer, V.; Schurig, V.

Tetrahedron: Asymmetry 2001, 12, 1395.

(60) Schmidt, C.; Straub, T.; Falabu, D.; Paulus, E. F.; Wegelius, E.;

Kolehmainen, E.; Bohmer, V.; Rissanen, K.; Vogt, W. Eur. J. Org. Chem. 2000, 3937.

(61) Elgihani, M. T.; Heaney, H.; Slawin, A. M. Z. Tetrahedron Lett. 1995, 36, 4905.

(62) Iwanek, W.; Mattay, J. Liebigs Annalen 1995, 1463; Iwanek, W.

Tetrahedron: Asymmetry 1998, 9, 4289.

(63) Smith, K. J.; Wilcox, J. D.; Mirick, G. E.; Wacker, L. S.; Ryan, N. S.; Vensel, D. A.; Readling, R.; Domush, H. L.; Amonoo, E. P.; Shariff, S. S.; Wenzel, T. J. Chirality 2003, 15, S150.

(64) Page, P. C. B.; Heaney, H.; Sampler, E. P. J. Am. Chem. Soc. 1999, 121, 6751 .

(65) Boxhall, J. Y.; Page, P. C. B.; Chan, Y.; Hayman, C. M.; Heaney, H.; McGrath, M. J. Synlett 2003, 997.

(66) Iwanek, W.; Froehlich, R.; Schwab, P.; Schurig, V. Chem. Commun.

(Cambridge, U. K.) 2002, 2516.

(67) Iwanek, W.; Urbaniak, M.; Gawdzik, B.; Schurig, V. Tetrahedron:

Asymmetry 2003, 14, 2787.

(68) Choi, H. J.; Buhring, D.; Quan, M. L. C.; Knobler, C. B.; Cram, D. J. J. Chem. Soc., Chem. Commun. 1992, 1733.

(69) Terent'eva, S. A.; Nikolaeva, I. L.; Burilov, A. R.; Kharitonov, D. I.; Popova, E. V.; Pudovik, M. A.; Litvinov, I. A.; Gubaidullin, A. T.; Konovalov, A. I. Russ. J. Gen. Chem. 2001, 71, 389.

(70) Lukin, O. V.; Pirozhenko, V. V.; Shivanyuk, A. N. Tetrahedron Lett. 1995, 36,7725 .

(71) McIldowie, M. J.; Mocerino, M.; Skelton, B. W.; White, A. H. Org. Lett. 2000, 2, 3869.

(72) Klaes, M.; Agena, C.; Kohler, M.; Inoue, M.; Wada, T.; Inoue, Y.; Mattay, J. Eur. J. Org. Chem. 2003, 1404.

(73) Klaes, M.; Neumann, B.; Stammler, H. G.; Mattay, J. Eur. J. Org. Chem. 2005, 864. 
(74) Buckley, B. R.; Page, P. C. B.; Chan, Y.; Heaney, H.; Klaes, M.; McIldowie, M. J.; McKee, V.; Mattay, J.; Mocerino, M.; Moreno, E.; Skelton, B. W.; White, A. H. Eur. J. Org. Chem. 2006, 5135. 